\title{
Serrin-Type Blowup Criterion for Viscous, Compressible, and Heat Conducting Navier-Stokes and Magnetohydrodynamic Flows
}

\author{
Xiangdi Huang, ${ }^{*} \quad$ Jing $\mathrm{Li}^{\dagger}$
}

\begin{abstract}
This paper establishes a blowup criterion for the three-dimensional viscous, compressible, and heat conducting magnetohydrodynamic (MHD) flows. It is essentially shown that for the Cauchy problem and the initial-boundary-value one of the three-dimensional compressible MHD flows with initial density allowed to vanish, the strong or smooth solution exists globally if the density is bounded from above and the velocity satisfies the Serrin's condition. Therefore, if the Serrin norm of the velocity remains bounded, it is not possible for other kinds of singularities (such as vacuum states vanish or vacuum appears in the non-vacuum region or even milder singularities) to form before the density becomes unbounded. This criterion is analogous to the well-known Serrin's blowup criterion for the threedimensional incompressible Navier-Stokes equations, in particular, it is independent of the temperature and magnetic field and is just the same as that of the barotropic compressible Navier-Stokes equations. As a direct application, it is shown that the same result also holds for the strong or smooth solutions to the three-dimensional full compressible Navier-Stokes system describing the motion of a viscous, compressible, and heat conducting fluid.
\end{abstract}

Keywords: compressible magnetohydrodynamic system, full compressible NavierStokes system, Serrin-type blowup criterion, vacuum.

AMS: 35Q35, 35B65, 76N10

\section{Introduction}

In this paper, we consider the system of partial differential equations for the threedimensional viscous, compressible, and heat conducting magnetohydrodynamic (MHD) flows in the Eulerian coordinates [21]

$$
\left\{\begin{array}{l}
\rho_{t}+\operatorname{div}(\rho u)=0, \\
(\rho u)_{t}+\operatorname{div}(\rho u \otimes u)-\mu \Delta u-(\mu+\lambda) \nabla \operatorname{div} u+\nabla P=(\operatorname{curl} H) \times H, \\
c_{v}\left[(\rho \theta)_{t}+\operatorname{div}(\rho u \theta)\right]-\kappa \Delta \theta+P \operatorname{div} u=2 \mu|\mathfrak{D}(u)|^{2}+\lambda(\operatorname{div} u)^{2}+\nu|\operatorname{curl} H|^{2}, \\
H_{t}-\operatorname{curl}(u \times H)=\nu \Delta H, \quad \operatorname{div} H=0,
\end{array}\right.
$$

*NCMIS, AMSS, Chinese Academy of Sciences, Beijing 100190, People's Republic of China; Department of Pure and Applied Mathematics, Graduate School of Information Science and Technology, Osaka University(xdhuang@amss.ac.cn). X. D. Huang is partially supported by NNSFC 11101392.

${ }^{\dagger}$ Institute of Applied Mathematics, AMSS, and Hua Loo-Keng Key Laboratory of Mathematics, Chinese Academy of Sciences, Beijing 100190, People's Republic of China (ajingli@gmail.com). J. $\mathrm{Li}$ is partially supported by the National Center for Mathematics and Interdisciplinary Sciences, CAS, and NNSFC 10971215 \& 11171326. 
where $t \geq 0$ is time, $x \in \Omega \subset \mathbb{R}^{3}$ is the spatial coordinate, and $\rho, u=\left(u_{1}, u_{2}, u_{3}\right)^{\operatorname{tr}}$, $\theta, P=R \rho \theta(R>0)$, and $H=\left(H_{1}, H_{2}, H_{3}\right)^{\mathrm{tr}}$, represent respectively the fluid density, velocity, absolute temperature, pressure, and magnetic field; $\mathfrak{D}(u)$ is the deformation tensor given by

$$
\mathfrak{D}(u)=\frac{1}{2}\left(\nabla u+(\nabla u)^{\mathrm{tr}}\right) .
$$

The constant viscosity coefficients $\mu$ and $\lambda$ satisfy the physical restrictions

$$
\mu>0, \quad 2 \mu+3 \lambda \geq 0 .
$$

Positive constants $c_{v}, \kappa$, and $\nu$ are respectively the heat capacity, the ratio of the heat conductivity coefficient over the heat capacity, and the magnetic diffusivity acting as a magnetic diffusion coefficient of the magnetic field.

The equations (1.1) will be studied with initial condition:

$$
(\rho, u, \theta, H)(x, 0)=\left(\rho_{0}, u_{0}, \theta_{0}, H_{0}\right)(x), \quad x \in \Omega,
$$

and one of the following boundary conditions:

1) If $\Omega=\mathbb{R}^{3}$, for constant $\tilde{\rho} \geq 0,(\rho, u, \theta, H)$ satisfies the far field condition:

$$
(\rho, u, H, \theta)(x, t) \rightarrow(\tilde{\rho}, 0,0,0) \text { as }|x| \rightarrow \infty
$$

2) If $\Omega$ is a bounded smooth domain in $\mathbb{R}^{3},(u, \theta, H)$ satisfies

$$
u=0, \quad \frac{\partial \theta}{\partial n}=0, \quad H=0 \quad \text { on } \partial \Omega,
$$

where $n=\left(n_{1}, n_{2}, n_{3}\right)$ is the unit outward normal to $\partial \Omega$.

The compressible MHD system (1.1) is a combination of the compressible NavierStokes equations of fluid dynamics and Maxwells equations of electromagnetism. Indeed, the equations $(1.1)_{1},(1.1)_{2}$, and $(1.1)_{3}$ describe, respectively, the conservation of mass, momentum, and energy. In addition, it is well-known that the electromagnetic fields are governed by Maxwells equations. In magnetohydrodynamics, the displacement current can be neglected ( [21]). As a consequence, the equation (1.1) 4 is called the induction equation, and the electric field can be written in terms of the magnetic field $H$ and the velocity $u$,

$$
E=\nu \nabla \times H-u \times H .
$$

Although the electric field $E$ does not appear in the compressible MHD system (1.1), it is indeed induced according to the above relation by the moving conductive flow in the magnetic field. In particular, when there is no electro-magnetic effect, that is, $H \equiv 0$, the compressible MHD system (1.1) reduces to the following full compressible NavierStokes system describing the motion of a viscous, compressible, and heat conducting fluid:

$$
\left\{\begin{array}{l}
\rho_{t}+\operatorname{div}(\rho u)=0, \\
(\rho u)_{t}+\operatorname{div}(\rho u \otimes u)-\mu \Delta u-(\mu+\lambda) \nabla \operatorname{div} u+\nabla P=0, \\
c_{v}\left[(\rho \theta)_{t}+\operatorname{div}(\rho u \theta)\right]-\kappa \Delta \theta+P \operatorname{div} u=2 \mu|\mathfrak{D}(u)|^{2}+\lambda(\operatorname{div} u)^{2} .
\end{array}\right.
$$

There is a considerable body of literature on the multi-dimensional full compressible Navier-Stokes system (1.6) and compressible MHD one (1.1) by physicists and mathematicians because of their physical importance, complexity, rich phenomena, and mathematical challenges; see [4, 5, 7, 8, 10, 11, 15, 21, 22, 24, 25, 27, 28, 36, 37] and the references 
cited therein. However, many physically important and mathematically fundamental problems are still open due to the lack of smoothing mechanism and the strong nonlinearity. For example, although the local strong solutions to the compressible MHD system (1.1) with large initial data were respectively obtained by [36] and [7] in the cases that the initial density is strictly positive and that the density is allowed to vanish initially, whether the unique local strong solution can exist globally is an outstanding challenging open problem.

Therefore, it is important to study the mechanism of blowup and structure of possible singularities of strong (or smooth) solutions to the compressible MHD system (1.1) and to the full compressible Navier-Stokes one (1.6). The pioneering work can be traced to Serrin's criterion [29] on the Leray-Hopf weak solutions to the three-dimensional incompressible Navier-Stokes equations, which can be stated that if a weak solution $u$ satisfies

$$
u \in L^{s}\left(0, T ; L^{r}\right), \quad \frac{2}{s}+\frac{3}{r} \leq 1, \quad 3<r \leq \infty,
$$

then it is regular. Later, He-Xin [9] showed that the Serrin's criterion (1.7) still holds even for the strong solution to the incompressible MHD equations.

Recently, Huang-Li-Xin [17] extended the Serrin's criterion (1.7) to the barotropic compressible Navier-Stokes equations and showed that if $T^{*}<\infty$ is the maximal time of existence of a strong (or classical) solution $(\rho, u)$, then

$$
\lim _{T \rightarrow T^{*}}\left(\|\operatorname{div} u\|_{L^{1}\left(0, T ; L^{\infty}\right)}+\|u\|_{L^{s}\left(0, T ; L^{r}\right)}\right)=\infty,
$$

and

$$
\lim _{T \rightarrow T^{*}}\left(\|\rho\|_{L^{\infty}\left(0, T ; L^{\infty}\right)}+\|u\|_{L^{s}\left(0, T ; L^{r}\right)}\right)=\infty,
$$

with $r$ and $s$ as in (1.7). For more information on the blowup criteria of barotropic compressible flow, we refer to [12, 13, 17, 18, 20, 34] and the references therein. Later $\mathrm{Xu}$-Zhang [38] extended the results of [17] to the isentropic compressible MHD system and obtained that the same blow-up criterion (1.9) holds.

When it comes to the full compressible Navier-Stokes system (1.6), the problem is much more complicated. Let $T^{*}<\infty$ be the maximal time of existence of a strong (or classical) solution $(\rho, u, \theta)$ to the system (1.6) . Besides (1.2), under the condition that

$$
7 \mu>\lambda
$$

Fan-Jiang-Ou [6] obtained that

$$
\lim _{T \rightarrow T^{*}}\left(\|\theta\|_{L^{\infty}\left(0, T ; L^{\infty}\right)}+\|\nabla u\|_{L^{1}\left(0, T ; L^{\infty}\right)}\right)=\infty .
$$

Recently, under just the physical restrictions (1.2), Huang-Li [14] and Huang-Li-Xin [18] established the following blowup criterion:

$$
\lim _{T \rightarrow T^{*}}\left(\|\theta\|_{L^{2}\left(0, T ; L^{\infty}\right)}+\|\mathfrak{D}(u)\|_{L^{1}\left(0, T ; L^{\infty}\right)}\right)=\infty
$$

where $\mathfrak{D}(u)$ is the deformation tensor. Later, in the absence of vacuum, Sun-WangZhang [35] showed that

$$
\lim _{T \rightarrow T^{*}}\left(\|\theta\|_{L^{\infty}\left(0, T ; L^{\infty}\right)}+\left\|\left(\rho, \rho^{-1}\right)\right\|_{L^{\infty}\left(0, T ; L^{\infty}\right)}\right)=\infty
$$


provided that (1.2) and (1.10) both hold. Very recently, under just the physical restrictions (1.2) and allowing the initial density to vanish, Huang-Li-Wang [16] improved all the previous results [6, 14, 18, 35] by obtaining that (1.8) still holds. It should be noted here that (1.9) is much stronger than (1.8) and that whether (1.9) holds or not remains open.

For the compressible MHD system (1.1), let $T^{*}<\infty$ be the maximal time of existence of a strong (or classical) solution $(\rho, u, \theta, H)$. Lu et al [23] obtained that

$$
\lim _{T \rightarrow T^{*}}\left(\|\rho\|_{L^{\infty}\left(0, T ; L^{\infty}\right)}+\|\theta\|_{L^{\infty}\left(0, T ; L^{\infty}\right)}+\|\nabla u\|_{L^{4}\left(0, T ; L^{2}\right)}\right)=\infty,
$$

and

$$
\lim _{T \rightarrow T^{*}}\left(\|\operatorname{div} u\|_{L^{\infty}\left(0, T ; L^{\infty}\right)}+\|\theta\|_{L^{\infty}\left(0, T ; L^{\infty}\right)}+\|\nabla u\|_{L^{4}\left(0, T ; L^{2}\right)}\right)=\infty
$$

while Chen-Liu [3] showed that

$$
\lim _{T \rightarrow T^{*}}\left(\|\nabla u\|_{L^{1}\left(0, T ; L^{\infty}\right)}+\|\theta\|_{L^{\infty}\left(0, T ; L^{\infty}\right)}\right)=\infty .
$$

The aim of this paper is to improve all the previous blowup criterion results on both the compressible MHD system (1.1) and the full compressible Navier-Stokes one (1.6) by allowing initial vacuum states, and by describing the blowup mechanism just in terms of the Serrin-type criterion, (1.9). Before stating our main result, we first explain the notations and conventions used throughout this paper. We denote

$$
\int f d x=\int_{\Omega} f d x
$$

For $1 \leq p \leq \infty$ and integer $k \geq 0$, the standard homogeneous and inhomogeneous Sobolev spaces are denoted by:

$$
\left\{\begin{array}{l}
L^{p}=L^{p}(\Omega), \quad W^{k, p}=W^{k, p}(\Omega), \quad D^{k, p}=\left\{u \in L_{\mathrm{loc}}^{1}(\Omega) \mid \nabla^{k} u \in L^{p}\right\}, \\
D_{0}^{1}=\left\{u \in L^{6} \mid \nabla u \in L^{2}, u=0 \text { on } \partial \Omega\right\}, \quad H_{0}^{1}=L^{2} \cap D_{0}^{1}, \quad H^{k}=W^{k, 2}, \\
D_{0, n}^{2,2}= \begin{cases}\left\{\theta \in D^{1,2} \cap D^{2,2} \mid \nabla \theta \cdot n=0 \text { on } \partial \Omega\right\}, & \text { for bounded } \Omega, \\
D_{0}^{1} \cap D^{2,2}, & \text { for } \Omega=\mathbb{R}^{3} .\end{cases}
\end{array}\right.
$$

Then, the strong solutions to the initial-boundary-value problem (1.1)-(1.3) together with (1.4) or (1.5) are defined as follows.

Definition 1.1 (Strong Solutions). For $\tilde{\rho} \geq 0$ and $\tilde{\theta}=0,(\rho, u, \theta, H)$ is called a strong solution to (1.1) in $\Omega \times(0, T)$, if for some $q_{0}>3$,

$$
\left\{\begin{array}{l}
\rho \geq 0, \quad \rho-\tilde{\rho} \in C\left([0, T] ; H^{1} \cap W^{1, q_{0}}\right), \quad \rho_{t} \in C\left([0, T] ; L^{2} \cap L^{q_{0}}\right), \\
(H, u) \in C\left([0, T] ; D_{0}^{1} \cap D^{2,2}\right) \cap L^{2}\left(0, T ; D^{2, q_{0}}\right), \quad H \in C\left([0, T] ; H^{2}\right) \\
\theta \geq 0, \quad \theta \in C\left([0, T] ; D_{0, n}^{2,2}\right) \cap L^{2}\left(0, T ; D^{2, q_{0}}\right), \\
\left(H_{t}, u_{t}, \theta_{t}\right) \in L^{2}\left(0, T ; D^{1,2}\right), \quad\left(H_{t}, \sqrt{\rho} u_{t}, \sqrt{\rho} \theta_{t}\right) \in L^{\infty}\left(0, T ; L^{2}\right),
\end{array}\right.
$$

and $(\rho, u, \theta, H)$ satisfies both (1.1) almost everywhere in $\Omega \times(0, T)$ and (1.3) almost everywhere in $\Omega$.

Our main result can be stated as follows: 
Theorem 1.1. For $\tilde{q} \in(3,6]$, assume that the initial data $\left(\rho_{0} \geq 0, u_{0}, \theta_{0} \geq 0, H_{0}\right)$ satisfies

$$
\begin{aligned}
& \rho_{0}-\tilde{\rho} \in H^{1} \cap W^{1, \tilde{q}}, \quad u_{0} \in D_{0}^{1} \cap D^{2,2}, \quad \theta_{0} \in D_{0, n}^{2,2}, \\
& \rho_{0}\left|u_{0}\right|^{4}+\rho_{0} \theta_{0}^{2} \in L^{1}, \quad H_{0} \in H_{0}^{1} \cap H^{2}, \quad \operatorname{div} H_{0}=0,
\end{aligned}
$$

and the compatibility conditions

$$
\begin{gathered}
-\mu \Delta u_{0}-(\mu+\lambda) \nabla \operatorname{div} u_{0}+R \nabla\left(\rho_{0} \theta_{0}\right)-\left(\operatorname{curl} H_{0}\right) \times H_{0}=\sqrt{\rho_{0}} g_{1}, \\
\kappa \Delta \theta_{0}+\frac{\mu}{2}\left|\nabla u_{0}+\left(\nabla u_{0}\right)^{t r}\right|^{2}+\lambda\left(\operatorname{div} u_{0}\right)^{2}+\nu\left|\operatorname{curl} H_{0}\right|^{2}=\sqrt{\rho_{0}} g_{2},
\end{gathered}
$$

with $g_{1}, g_{2} \in L^{2}$. Let $(\rho, u, \theta, H)$ be the strong solution to the initial boundary value problem (1.1) -(1.3) together with (1.4) or (1.5). If $T^{*}<\infty$ is the maximal time of existence, then for $r$ and $s$ as in (1.7),

$$
\lim _{T \rightarrow T^{*}}\left(\|\rho\|_{L^{\infty}\left(0, T ; L^{\infty}\right)}+\|u\|_{L^{s}\left(0, T ; L^{r}\right)}\right)=\infty .
$$

If $H \equiv H_{0} \equiv 0$, Theorem 1.1 directly yields the following Serrin-type blowup criterion for the three-dimensional full compressible Navier-Stokes system (1.6).

Theorem 1.2. For constants $\tilde{q} \in(3,6]$ and $\tilde{\rho} \geq 0$, assume that $\left(\rho_{0} \geq 0, u_{0}, \theta_{0} \geq 0\right)$ satisfies

$$
\rho_{0}-\tilde{\rho} \in H^{1} \cap W^{1, \tilde{q}}, \quad u_{0} \in D_{0}^{1} \cap D^{2,2}, \quad \theta_{0} \in D_{0, n}^{2,2}, \quad \rho_{0}\left|u_{0}\right|^{4}+\rho_{0} \theta_{0}^{2} \in L^{1},
$$

and the compatibility conditions

$$
\begin{aligned}
-\mu \Delta u_{0}-(\mu+\lambda) \nabla \operatorname{div} u_{0}+R \nabla\left(\rho_{0} \theta_{0}\right) & =\sqrt{\rho_{0}} g_{1}, \\
\kappa \Delta \theta_{0}+\frac{\mu}{2}\left|\nabla u_{0}+\left(\nabla u_{0}\right)^{t r}\right|^{2}+\lambda\left(\operatorname{div} u_{0}\right)^{2} & =\sqrt{\rho_{0}} g_{2},
\end{aligned}
$$

with $g_{1}, g_{2} \in L^{2}$. Let $(\rho, u, \theta)$ be the strong solution to the full compressible NavierStokes system (1.6) together with

$$
(\rho, u, \theta)(x, 0)=\left(\rho_{0}, u_{0}, \theta_{0}\right), \quad x \in \Omega,
$$

and either for $\Omega=\mathbb{R}^{3}$,

$$
(\rho, u, \theta) \rightarrow(\tilde{\rho}, 0,0) \text { as }|x| \rightarrow \infty
$$

or for a bounded smooth domain $\Omega \subset \mathbb{R}^{3}$,

$$
u=0, \quad \frac{\partial \theta}{\partial n}=0 \text { on } \partial \Omega
$$

If $T^{*}<\infty$ is the maximal time of existence, then

$$
\lim _{T \rightarrow T^{*}}\left(\|\rho\|_{L^{\infty}\left(0, T ; L^{\infty}\right)}+\|u\|_{L^{s}\left(0, T ; L^{r}\right)}\right)=\infty
$$

with $r$ and $s$ as in (1.7).

A few remarks are in order:

Remark 1.1. The conclusion in Theorem 1.1 is somewhat surprising since the criterion (1.14) is independent of the temperature and magnetic fields and just the same as those of barotropic compressible Navier-Stokes equations ( [17]). 
Remark 1.2. In [16, Theorem 1], we obtained that (1.8) holds for the Cauchy problem of the full compressible Navier-Stokes system (1.6). Thus,

$$
\lim _{T \rightarrow T^{*}}\|\operatorname{div} u\|_{L^{1}\left(0, T ; L^{\infty}\right)}=\infty
$$

provided that

$$
\sup _{0 \leq T \leq T^{*}}\|u\|_{L^{r}\left(0, T ; L^{s}\right)}<\infty
$$

for $r, s$ as in (1.7). It follows from the continuity equation (1.6) 1 that for $t \in\left[0, T^{*}\right)$,

$$
\rho(x, t)=\rho_{0}(y(0 ; x, t)) \exp \left\{-\int_{0}^{t} \operatorname{div} u(y(s ; x, t), s) d s\right\},
$$

where $y(s ; x, t)$ is the characteristic curve defined by

$$
\frac{\mathrm{d}}{\mathrm{d} s} y=u(y, s), \quad y(t ; x, t)=x .
$$

The combination of (1.19) with (1.21) implies that there may hold for the density:

1) The density remains bounded, that is,

$$
\lim _{T \rightarrow T^{*}}\|\rho\|_{L^{\infty}\left(0, T ; L^{\infty}\right)}<\infty
$$

2) The density may concentrate, that is,

$$
\lim _{T \rightarrow T^{*}}\|\rho\|_{L^{\infty}\left(0, T ; L^{\infty}\right)}=\infty
$$

3) Vacuum states may vanish: There exists some $x_{1} \in \Omega$ and $x_{1}(t)$ satisfying $\rho_{0}\left(x_{1}\right)=0$ and $y\left(0 ; x_{1}(t), t\right)=x_{1}$ such that

$$
\lim _{t \rightarrow T^{*}} \rho\left(x_{1}(t), t\right) \geq c_{0}>0
$$

4) Vacuum states may appear in the non-vacuum region: There exists some $x_{2} \in \Omega$ and $x_{2}(t)$ satisfying $\rho_{0}\left(x_{2}\right)>0$ and $y\left(0 ; x_{2}(t), t\right)=x_{2}$ such that

$$
\lim _{t \rightarrow T^{*}} \rho\left(x_{2}(t), t\right)=0 .
$$

Then one may ask: Which one or some of (1.22)-(1.25) will happen? Theorem 1.2 gives an answer to this question by obtaining that the density will concentrate provided that (1.20) holds. In other words, if the Serrin norm of the velocity remains bounded, it is not possible for other kinds of singularities (such as vacuum states vanish or vacuum appears in the non-vacuum region or even milder singularities) to form before the density becomes unbounded. Moreover, (1.18) still holds for the initial-boundaryvalue problem (1.6) (1.15) (1.17). Thus, Theorem 1.2 greatly improves all the previous blowup criterion for the full compressible Navier-Stokes system (1.6) [6, 13, 14, 16, 35].

Remark 1.3. If $\Omega$ is a bounded smooth domain of $\mathbb{R}^{3}$, Theorems 1.1 and 1.2 still hold when the boundary condition $\left.\nabla \theta \cdot n\right|_{\partial \Omega}=0$ is replaced by $\left.\theta\right|_{\partial \Omega}=0$.

Remark 1.4. Theorems 1.1 and 1.2 also hold respectively for classical solutions to the three-dimensional compressible MHD system (1.1) and to the full compressible NavierStokes one (1.6). 
We now comment on the analysis of this paper.

Let $(\rho, u, \theta, H)$ be a strong solution described in Theorem 1.1. Suppose that (1.14) were false, that is,

$$
\lim _{T \rightarrow T^{*}}\left(\|\rho\|_{L^{\infty}\left(0, T ; L^{\infty}\right)}+\|u\|_{L^{s}\left(0, T ; L^{r}\right)}\right) \leq M_{0}<+\infty .
$$

We want to show that

$$
\sup _{0 \leq t \leq T^{*}}\left(\|\rho-\tilde{\rho}\|_{H^{1} \cap W^{1, \tilde{q}}}+\|\nabla u\|_{H^{1}}+\|\nabla \theta\|_{H^{1}}+\|H\|_{H^{2}}\right) \leq C<+\infty .
$$

Since the methods in all previous works $[3,6,16,23,35,35]$ depend crucially on either the $L_{t}^{\infty} L_{x}^{\infty}$-norm of the temperature $\theta$ or the $L_{t}^{1} L_{x}^{\infty}$-norm of the divergence of the velocity $\operatorname{div} u$, some new ideas are needed to recover all the a priori estimates just under the assumption (1.26) without any a priori bounds on the temperature, the magnetic field, and the divergence of the velocity. In fact, we prove (see Lemma 3.3) that a control of the Serrin norm of the velocity and $L_{t}^{\infty} L_{x}^{\infty}$-norm of the density implies a control on the $L_{t}^{\infty} L_{x}^{2}$ norm of $\nabla u$. In order to obtain this control, the key observation is that, instead of the temperature $\theta$, we treat the total energy $E=c_{v} \theta+\frac{1}{2}|u|^{2}$, which in turn greatly reduces the difficulties arising from the high nonlinearities of the temperature equation, (1.1) 3 . Indeed, multiplying the equation of the conservative form of the total energy $E$ (see (3.5)) by $E$ yields that to bound the $L_{t}^{2} L_{x}^{2}$-norm of $\nabla E$ (see (3.4)), it is enough to control that of $|u||\nabla u|$, which in fact can be reduced to the estimate of the $L_{t}^{2} L_{x}^{6}$-norm of $\nabla u$ (see (3.30) $)$. Then, to overcome the difficulty caused by the boundary when $\Omega$ is bounded, motivated by [12, 34, we decompose the velocity into two parts (see (3.14) and (3.18) ) which together with the $L^{p}$-estimate for the Lamé system yield the desired bound on the $L_{t}^{2} L_{x}^{6}$-norm of $\nabla u$ (see (3.31) ). Finally, the a priori estimates on both the $L_{t}^{\infty} L_{x}^{p}$-norm of the density gradient and the $L_{t}^{1} L_{x}^{\infty}$-norm of the velocity gradient can be obtained simultaneously by solving a logarithm Gronwall inequality based on a logarithm estimate for the Lamé system (see Lemma 2.3) and the a priori estimates we have just derived.

The rest of the paper is organized as follows: In the next section, we collect some elementary facts and inequalities that will be needed later. The main result, Theorem 1.1, is proved in Section 3 .

\section{Preliminaries}

In this section, we recall some known facts and elementary inequalities that will be used later.

First, the following existence and uniqueness of local strong solutions when the initial density may not be positive and may vanish in an open set can be proved in a similar way as in [4] (cf. [7]).

Lemma 2.1. Assume that the initial data $\left(\rho_{0} \geq 0, u_{0}, \theta_{0} \geq 0, H_{0}\right)$ satisfy (1.11)-(1.13). Then there exists a positive time $T_{1} \in(0, \infty)$ and a unique strong solution $(\rho, u, \theta, H)$ to the initial-boundary-value problem (1.1) -(1.3) together with (1.4) or (1.5) on $\Omega \times\left(0, T_{1}\right]$.

Next, the following well-known Sobolev inequality will be used later frequently (see [26]). 
Lemma 2.2. For $p \in(1, \infty)$ and $q \in(3, \infty)$, there exists a generic constant $C>0$, which depends only on $p, q$ such that for $f \in D_{0}^{1}$ and $g \in L^{p} \cap D^{1, q}$, we have

$$
\|f\|_{L^{6}} \leq C\|\nabla f\|_{L^{2}}, \quad\|g\|_{L^{\infty}} \leq C\|g\|_{L^{p}}+C\|\nabla g\|_{L^{q}} .
$$

Finally, we consider the following Lamé system

$$
-\mu \Delta v(x)-(\mu+\lambda) \nabla \operatorname{div} v(x)=f(x), \quad x \in \Omega,
$$

where $v=\left(v_{1}, v_{2}, v_{3}\right), f=\left(f_{1}, f_{2}, f_{3}\right)$, and $\mu, \lambda$ satisfy (1.2). The system (2.2) is imposed on one of the following boundary conditions:

1) Cauchy problem: $\Omega=\mathbb{R}^{3}$, and

$$
v(x) \rightarrow 0, \text { as }|x| \rightarrow \infty
$$

2) Dirichlet problem: $\Omega$ is a bounded smooth domain in $\mathbb{R}^{3}$, and

$$
v=0 \text { on } \partial \Omega \text {. }
$$

The following logarithm estimate for the Lamé system (2.2) will be used to estimate $\|\nabla u\|_{L^{\infty}}$ and $\|\nabla \rho\|_{L^{2} \cap L^{q}}$.

Lemma 2.3. Let $\mu, \lambda$ satisfy (1.2). Assume that $f=\operatorname{div} g$ where $g=\left(g_{k j}\right)_{3 \times 3}$ with $g_{k j} \in L^{2} \cap L^{r} \cap D^{1, q}$ for $k, j=1, \cdots, 3, r \in(1, \infty)$, and $q \in(3, \infty)$. Then the Lamé system (2.2) together with (2.3) or (2.4) has a unique solution $v \in D_{0}^{1} \cap D^{1, r} \cap D^{2, q}$, and there exists a generic positive constant $C$ depending only on $\mu, \lambda, q$, and $r$ (besides $\Omega$ when $\Omega$ is bounded) such that

$$
\|\nabla v\|_{L^{r}} \leq C\|g\|_{L^{r}}
$$

and

$$
\|\nabla v\|_{L^{\infty}} \leq C\left(1+\ln \left(e+\|\nabla g\|_{L^{q}}\right)\|g\|_{L^{\infty}}+\|g\|_{L^{r}}\right) .
$$

Proof. First, if $\Omega=\mathbb{R}^{3}$, direct calculations show that $v=\left(v_{1}, v_{2}, v_{3}\right)$ with

$$
v_{j}=\frac{1}{2 \mu+\lambda}(-\Delta)^{-1} \partial_{k} g_{k j}, \quad j=1, \cdots, 3
$$

is the unique solution to the Cauchy problem (2.2) (2.3) and satisfies (2.5).

Then, if $\Omega$ is a bounded smooth domain of $\mathbb{R}^{3}$, it follows from 33 . that the Dirichlet problem (2.2) (2.4) is of Petrovsky type. In Petrovsky's systems, roughly speaking, different equations and unknowns have the same "differentiability order", see [32, p.126]. We also recall that Petrovsky's systems are an important subclass of Agmon-DouglisNirenberg (ADN) elliptic systems( [1]), having the same good properties of self-adjoint ADN systems. It follows from Solonnikov [32, Theorem 1.1] and [33, Theorem 5.1] that the solution $v$ to the system (2.2) together with (2.4) can be represented as

$$
v_{i}(x)=\int G_{i j}(x, y) f_{j}(y) d y, \quad \text { for all } x \in \Omega,
$$

by means of the Green function $G_{i j}=G_{i j}(x, y) \in C^{\infty}(\Omega \times \Omega \backslash D)$ with $D \equiv\{(x, y) \in$ $\Omega \times \Omega \mid x=y\}$ which satisfies that for every multi-indexes $\alpha=\left(\alpha_{1}, \alpha_{2}, \alpha_{3}\right)$ and $\beta=$ $\left(\beta_{1}, \beta_{2}, \beta_{3}\right)$ there is a constant $C_{\alpha, \beta}$ such that for all $(x, y) \in \Omega \times \Omega \backslash D$, and $i, j=1, \cdots, 3$,

$$
\left|\partial_{x}^{\alpha} \partial_{y}^{\beta} G_{i j}(x, y)\right| \leq C_{\alpha, \beta}|x-y|^{-1-|\alpha|-|\beta|},
$$


where $|\alpha|=\alpha_{1}+\alpha_{2}+\alpha_{3}$ and $\beta=\beta_{1}+\beta_{2}+\beta_{3}$. Moreover, the estimate (2.5) is standard.

Finally, it remains to prove (2.6). We will only deal with the Dirichlet problem (2.2) (2.4), since the same procedure holds for the Cauchy problem (2.2) (2.3). Motivated by Beale-Kato-Majda [2], we introduce a small parameter $\delta \in(0,1]$ which depends on $v$, and which will be fixed later. Using $\delta$, we define a cut-off function $\eta_{\delta}(s)$ satisfying $\eta_{\delta}(s)=1$ for $0 \leq s<\delta, \eta_{\delta}(s)=0$ for $s>2 \delta$, and $\left|\eta_{\delta}^{(k)}(s)\right| \leq C \delta^{-k}$. It thus follows from (2.7) that

$$
\begin{aligned}
v_{i}(x)= & \int\left(\eta_{\delta}(|x-y|)+\left(1-\eta_{\delta}(|x-y|)\right)\right) G_{i j}(x, y) \partial_{k} g_{k j}(y) d y \\
= & \int \eta_{\delta}(|x-y|) G_{i j}(x, y) \partial_{k} g_{k j}(y) d y+\int \partial_{y_{k}} \eta_{\delta}(|x-y|) G_{i j}(x, y) g_{k j}(y) d y \\
& -\int\left(1-\eta_{\delta}(|x-y|)\right) \partial_{y_{k}} G_{i j}(x, y) g_{k j}(y) d y
\end{aligned}
$$

where in the second equality we have used integrations by parts due to the fact that $\left.G_{i j}(x, y)\right|_{\partial \Omega}=0$ for each $x \in \Omega$. Hence, we have

$$
\begin{aligned}
|\nabla v(x)| \leq & C \int\left(\left|\eta_{\delta}^{\prime}\right|\left|G_{i j}\right|+\eta_{\delta}\left|\nabla_{x} G_{i j}\right|\right)|\nabla g| d y \\
& +C \int\left(\left|\eta_{\delta}^{\prime \prime}\right|\left|G_{i j}\right|+\left|\eta_{\delta}^{\prime}\right|\left|\nabla_{x} G_{i j}\right|+\left|\eta_{\delta}^{\prime}\right|\left|\nabla_{y} G_{i j}\right|\right)|g| d y \\
& +C \int\left(1-\eta_{\delta}\right)\left|\nabla_{x} \nabla_{y} G_{i j}\right||g| d y
\end{aligned}
$$

Each term on the right-hand side of (2.9) can be estimated by (2.8) as follows:

$$
\begin{aligned}
& \int\left(\left|\eta_{\delta}^{\prime}\right|\left|G_{i j}\right|+\eta_{\delta}\left|\nabla_{x} G_{i j}\right|\right)|\nabla g| d y \\
& \leq C \int_{\Omega \cap\{y|| x-y \mid<2 \delta\}}\left(\delta^{-1}|x-y|^{-1}+|x-y|^{-2}\right)|\nabla g| d y \\
& \leq C\left(\delta^{-q /(q-1)} \int_{0}^{2 \delta} s^{-q /(q-1)} s^{2} d s+\int_{0}^{2 \delta} s^{-2 q /(q-1)} s^{2} d s\right)^{(q-1) / q}\|\nabla g\|_{L^{q}} \\
& \leq C \delta^{(q-3) / q}\|\nabla g\|_{L^{q}}, \\
& \int\left(\left|\eta_{\delta}^{\prime \prime}\right|\left|G_{i j}\right|+\left|\eta_{\delta}^{\prime}\right|\left|\nabla_{x} G_{i j}\right|+\left|\eta_{\delta}^{\prime}\right|\left|\nabla_{y} G_{i j}\right|\right)|g| d y \\
& \leq C \int_{\delta}^{2 \delta}\left(\delta^{-2} s^{-1}+\delta^{-1} s^{-2}\right) s^{2} d s\|g\|_{L^{\infty}} \\
& \leq C\|g\|_{L^{\infty}}
\end{aligned}
$$

and

$$
\begin{aligned}
& \int\left(1-\eta_{\delta}\right)\left|\nabla_{x} \nabla_{y} G_{i j}\right||g| d y \\
& \leq C\left(\int_{\Omega \cap\{y|\delta \leq| x-y \mid \leq 1\}}+\int_{\Omega \cap\{y|| x-y \mid>1\}}\right)|x-y|^{-3}|g(y)| d y \\
& \leq C \int_{\delta}^{1} s^{-3} s^{2} d s\|g\|_{L^{\infty}}+C\left(\int_{1}^{\infty} s^{-3 r /(r-1)} s^{2} d s\right)^{(r-1) / r}\|g\|_{L^{r}} \\
& \leq-C\|\|_{L^{\infty}} \ln \delta+C\|g\|_{L^{r}} .
\end{aligned}
$$


It follows from (2.9)-(2.12) that

$$
\|\nabla v\|_{L^{\infty}} \leq C\left(\delta^{(q-3) / q}\|\nabla g\|_{L^{q}}+(1-\ln \delta)\|g\|_{L^{\infty}}+\|g\|_{L^{r}}\right) .
$$

Set $\delta=\min \left\{1,\|\nabla g\|_{L^{q}}^{-q /(q-3)}\right\}$. Then (2.13) becomes (2.6). We finish the proof of Lemma 2.3 .

\section{Proof of Theorem 1.1}

Before proving Theorem 1.1, we state some a priori estimates under the condition (1.26). First, we have

Lemma 3.1. Under the condition (1.26), it holds that for $q \in[2,12]$ and $0 \leq T<T^{*}$,

$$
\|H\|_{L^{\infty}\left(0, T ; L^{q}\right)}+\int_{0}^{T} \int|H|^{q-2}|\nabla H|^{2} d x \leq C,
$$

where (and in what follows) $C$ and $C_{i}(i=1, \cdots, 6)$ denote generic constants depending only on $M_{0}, \mu, \lambda, R, \kappa, c_{v}, T^{*}, \nu$, and the initial data (besides $\Omega$ for bounded $\Omega$ ).

Proof. Similar to [38], multiplying $(1.1)_{4}$ by $q|H|^{q-2} H$ and integrating the resulting equation over $\Omega$ yield that

$$
\begin{aligned}
\frac{d}{d t} \int|H|^{q} d x+\nu \int\left(q|H|^{q-2}|\nabla H|^{2}+\left.q(q-2)|H|^{q-2}|\nabla| H\right|^{2}\right) d x \\
=-\int q|H|^{q-2}\left(H \cdot \nabla H \cdot u-\frac{q-1}{2} u \cdot \nabla|H|^{2}\right) d x \\
\quad-\frac{q(q-2)}{2} \int|H|^{q-4}\left(H \cdot \nabla|H|^{2}\right)(u \cdot H) d x \\
\leq \frac{\nu}{2} \int q|H|^{q-2}|\nabla H|^{2} d x+C q^{2} \int|u|^{2}|H|^{q} d x \\
\leq \frac{\nu}{2} \int q|H|^{q-2}|\nabla H|^{2} d x+C\|u\|_{L^{r}}^{2}\left\||H|^{q / 2}\right\|_{L^{2}}^{2(r-3) / r}\left\||H|^{q / 2}\right\|_{L^{6}}^{6 / r} \\
\leq \frac{\nu}{2} \int q|H|^{q-2}|\nabla H|^{2} d x+C \delta\left\|\nabla|H|^{q / 2}\right\|_{L^{2}}^{2}+C(\delta)\left(1+\|u\|_{L^{r}}^{s}\right)\|H\|_{L^{q}}^{q} .
\end{aligned}
$$

Choosing $\delta$ suitably small in (3.2), we obtain (3.1) directly after using Gronwall's inequality and (1.26). We thus finish the proof of Lemma 3.1 .

Then, we derive the following key estimate on the specific energy $E$ defined by

$$
E \triangleq c_{v} \theta+\frac{|u|^{2}}{2}
$$

Lemma 3.2. Under the condition (1.26), it holds that

$$
\begin{gathered}
c_{v} \frac{d}{d t} \int \rho E^{2} d x+\kappa\|\nabla E\|_{L^{2}}^{2} \leq \\
C \int|u|^{2}\left(\rho E^{2}+|\nabla u|^{2}\right) d x+C_{1}\|\nabla H\|_{H^{1}}^{2} \\
+C\|\nabla u\|_{L^{2}}^{2}+C \int \rho E^{2} d x .
\end{gathered}
$$


Proof. First, it follows from (1.1) that $E$ satisfies

$$
(\rho E)_{t}+\operatorname{div}(\rho E u)-\frac{\kappa}{c_{v}} \Delta E=\operatorname{div} F-H^{i} H^{j} \partial_{i} u^{j}+\frac{1}{2}|H|^{2} \operatorname{div} u+\nu|\operatorname{curl} H|^{2},
$$

with

$$
F \triangleq \frac{\mu-\kappa c_{v}^{-1}}{2} \nabla\left(|u|^{2}\right)+\mu u \cdot \nabla u+\lambda u \operatorname{div} u-P u+(u \cdot H) H-\frac{1}{2}|H|^{2} u .
$$

Next, applying standard maximum principle to $(1.1)_{3}$ together with $\theta_{0} \geq 0$ (c.f. [6, 8] ) shows

$$
\inf _{\mathbb{R}^{3} \times[0, T]} \theta(x, t) \geq 0
$$

Multiplying (3.5) by $c_{v} E$ and integrating the resulting equality over $\Omega$, we obtain after integration by parts and using (1.1 $)_{1}$ that

$$
\begin{aligned}
& \frac{c_{v}}{2} \frac{d}{d t} \int \rho E^{2} d x+\kappa \int|\nabla E|^{2} d x \\
& \leq C \int(|u||\nabla u|+\rho \theta|u|)|\nabla E| d x+C \int\left(|u||H|^{2}|\nabla E|+|\nabla u||H|^{2} E\right) d x \\
& \quad+C \int E|\operatorname{curl} H|^{2} d x .
\end{aligned}
$$

We estimate each term on the right-hand side of (3.6) as follows:

First, Holder's inequality gives

$$
\int(|u||\nabla u|+\rho \theta|u|)|\nabla E| d x \leq \eta\|\nabla E\|_{L^{2}}^{2}+C(\eta) \int\left(|u|^{2}|\nabla u|^{2}+\rho E^{2}|u|^{2}\right) d x .
$$

Next, if $\Omega=\mathbb{R}^{3}$, Sobolev's inequality gives that there exists a universal constant $C$ such that

$$
\|E\|_{L^{6}} \leq C\|\nabla E\|_{L^{2}} .
$$

If $\Omega$ is a bounded smooth domain in $\mathbb{R}^{3}$, the Poincaré-type inequality ( $[\underline{8}$, Lemma 3.2]) shows there exists a generic positive constant $C$ which also depends on $\Omega$ such that

$$
\|E\|_{L^{6}} \leq C\left\|\rho^{1 / 2} E\right\|_{L^{2}}+C\|\nabla E\|_{L^{2}},
$$

which combined with (3.8) implies

$$
\|E\|_{L^{6}} \leq C\left\|\rho^{1 / 2} E\right\|_{L^{2}}+C\|\nabla E\|_{L^{2}} .
$$

It thus follows from Holder's inequality, (3.9), (3.1), and (2.1) that

$$
\begin{aligned}
& \int\left(|u||H|^{2}|\nabla E|+|\nabla u \| H|^{2} E\right) d x \\
& \leq\|u\|_{L^{6}}\|H\|_{L^{6}}^{2}\|\nabla E\|_{L^{2}}+\|\nabla u\|_{L^{2}}\|H\|_{L^{6}}^{2}\|E\|_{L^{6}} \\
& \leq \eta\|\nabla E\|_{L^{2}}^{2}+C(\eta)\left\|\rho^{1 / 2} E\right\|_{L^{2}}^{2}+C(\eta)\|\nabla u\|_{L^{2}}^{2} .
\end{aligned}
$$

Finally, integration by parts together with (3.9) yields

$$
\begin{aligned}
\int E|\operatorname{curl} H|^{2} d x & \leq C \int|\nabla E\|\nabla H\| H| d x+C \int\left|E\left\|\nabla^{2} H\right\| H\right| d x \\
& \leq C\|\nabla E\|_{L^{2}}\|\nabla H\|_{L^{6}}\|H\|_{L^{3}}+C\|E\|_{L^{6}}\left\|\nabla^{2} H\right\|_{L^{2}}\|H\|_{L^{3}} \\
& \leq \eta\|\nabla E\|_{L^{2}}^{2}+C(\eta)\left\|\rho^{1 / 2} E\right\|_{L^{2}}^{2}+C(\eta)\|\nabla H\|_{H^{1}}^{2}
\end{aligned}
$$


Putting (3.7), (3.10), and (3.11) into (3.6), we obtain (3.4) after choosing $\eta$ suitably small. The proof of Lemma 3.2 is completed.

Then, we derive the following crucial estimate on the $L^{\infty}\left(0, T ; L^{2}\right)$-norm of $\nabla u$.

Lemma 3.3. Under the condition (1.26), it holds that for $0 \leq T<T^{*}$,

$$
\begin{aligned}
& \sup _{0 \leq t \leq T} \int\left((\rho-\tilde{\rho})^{2}+\rho \theta^{2}+|\nabla u|^{2}+|\nabla H|^{2}\right) d x \\
& +\int_{0}^{T} \int\left(|\nabla \theta|^{2}+\rho|\dot{u}|^{2}+\left|H_{t}\right|^{2}+\left|\nabla^{2} H\right|^{2}\right) d x d t \leq C .
\end{aligned}
$$

Proof. First, multiplying $(1.1)_{2}$ by $u_{t}$ and integrating the resulting equation over $\Omega$ show that

$$
\begin{aligned}
& \frac{1}{2} \frac{d}{d t} \int\left(\mu|\nabla u|^{2}+(\mu+\lambda)(\operatorname{div} u)^{2}\right) d x+\int \rho|\dot{u}|^{2} d x \\
& =\int \rho \dot{u} \cdot(u \cdot \nabla) u d x+\int P \operatorname{div} u_{t} d x-\frac{1}{2} \int\left(\nabla|H|^{2}-2 \operatorname{div}(H \otimes H)\right) \cdot u_{t} d x \\
& \leq \frac{1}{4} \int \rho|\dot{u}|^{2} d x+C \int \rho|u|^{2}|\nabla u|^{2} d x+\frac{d}{d t} \int P \operatorname{div} u d x \\
& \quad-\int P_{t} \operatorname{div} u d x+\frac{1}{2} \int\left(|H|^{2} \operatorname{div} u_{t}-2 H \cdot \nabla u_{t} \cdot H\right) d x .
\end{aligned}
$$

Then, we will estimate the last two terms on the right-hand side of (3.13).

On the one hand, to overcome the difficulty caused by the boundary, motivated by [12,34, we decompose the velocity into two parts. It follows from Lemma 2.3 that for any $t \in[0, T]$, there exists a unique $v(t, \cdot) \in D_{0}^{1} \cap D^{2,2} \cap D^{2, \tilde{q}}$ satisfying

$$
\mu \triangle v+(\mu+\lambda) \nabla \operatorname{div} v=\nabla P,
$$

which together with (2.5) yields that

$$
\|\nabla v\|_{L^{p}} \leq C\|P\|_{L^{p}} \leq C\|\rho E\|_{L^{p}}, \text { for } p \in[2,6], t \in[0, T],
$$

and that

$$
\begin{aligned}
-\int P_{t} \operatorname{div} v d x & =-\int\left(\mu \nabla v_{t} \cdot \nabla v+(\mu+\lambda) \operatorname{div} v_{t} \operatorname{div} v\right) d x \\
& =-\frac{1}{2} \frac{d}{d t} \int\left(\mu|\nabla v|^{2}+(\mu+\lambda)(\operatorname{div} v)^{2}\right) d x
\end{aligned}
$$

Denoting by

$$
w \triangleq u-v
$$

we have $w \in D_{0}^{1} \cap D^{2,2} \cap D^{2, \tilde{q}}$, for a.e. $t \in[0, T]$. Moreover, for a.e. $t \in[0, T], w$ satisfies

$$
\mu \triangle w+(\mu+\lambda) \nabla \operatorname{div} w=\rho \dot{u}+H \times(\operatorname{curl} H),
$$

which together with the standard $L^{2}$-estimate for elliptic system gives

$$
\begin{aligned}
\|\nabla w\|_{L^{6}}+\left\|\nabla^{2} w\right\|_{L^{2}} & \leq C\|\rho \dot{u}\|_{L^{2}}+C\|\| H\|\nabla H \mid\|_{L^{2}} \\
& \leq C\|\rho \dot{u}\|_{L^{2}}+C\|H\|_{L^{6}}\|\nabla H\|_{L^{2}}^{1 / 2}\|\nabla H\|_{L^{6}}^{1 / 2} \\
& \leq C\|\rho \dot{u}\|_{L^{2}}+C\|\nabla H\|_{L^{2}}^{1 / 2}\|\nabla H\|_{H^{1}}^{1 / 2}
\end{aligned}
$$


due to (3.1). It follows from (3.3) and (3.5) that

$$
\begin{aligned}
-\int P_{t} \operatorname{div} w d x= & -\frac{R}{c_{v}} \int(\rho E)_{t} \operatorname{div} w d x+\frac{R}{2 c_{v}} \int\left(\rho|u|^{2}\right)_{t} \operatorname{div} w d x \\
\leq & C \int\left(\rho E|u|+|\nabla E|+|u||\nabla u|+|u||H|^{2}\right)\left|\nabla^{2} w\right| d x \\
& +C \int|H|^{2}|\nabla u||\nabla w| d x-\frac{R \nu}{c_{v}} \int|\operatorname{curl} H|^{2} \operatorname{div} w d x \\
& -\frac{R}{2 c_{v}} \int\left(\operatorname{div}(\rho u)|u|^{2} \operatorname{div} w-2 \rho u \cdot u_{t} \operatorname{div} w\right) d x=\sum_{i=1}^{4} I_{i} .
\end{aligned}
$$

Cauchy's and Sobolev's inequalities together with (3.1) yield that

$$
\begin{aligned}
I_{1}+I_{2} \leq & \eta\left(\left\|\nabla^{2} w\right\|_{L^{2}}^{2}+\|\nabla w\|_{L^{6}}^{2}\right) \\
& +C(\eta) \int\left(\rho^{2} E^{2}|u|^{2}+|\nabla E|^{2}+|u|^{2}|\nabla u|^{2}+|\nabla u|^{2}\right) d x .
\end{aligned}
$$

Similar to (3.11), integration by parts leads to

$$
\begin{aligned}
I_{3} & \leq C \int\left(\left|\nabla H\|H\| \nabla^{2} w\right|+\left|\nabla^{2} H\|H\| \nabla w\right|\right) d x \\
& \leq C\left(\|\nabla H\|_{L^{6}}\left\|\nabla^{2} w\right\|_{L^{2}}+\left\|\nabla^{2} H\right\|_{L^{2}}\|\nabla w\|_{L^{6}}\right)\|H\|_{L^{3}} \\
& \leq \eta\left(\left\|\nabla^{2} w\right\|_{L^{2}}^{2}+\|\nabla w\|_{L^{6}}^{2}\right)+C(\eta)\|\nabla H\|_{H^{1}}^{2},
\end{aligned}
$$

where in the last inequality we have used (3.1).

Integration by parts also gives

$$
\begin{aligned}
I_{4} \leq & C \int \rho|u|^{3}\left|\nabla^{2} w\right| d x+C \int\left(\rho|u|^{2}|\nabla u|+\rho|u||\dot{u}|\right)|\nabla w| d x \\
\leq & C(\eta) \int\left(\rho E^{2}|u|^{2}+\rho|u|^{2}|\nabla u|^{2}+\rho|u|^{2}|\nabla v|^{2}\right) d x \\
& +\eta\left\|\nabla^{2} w\right\|_{L^{2}}^{2}+\eta \int \rho|\dot{u}|^{2} d x .
\end{aligned}
$$

On the other hand, direct calculations show

$$
\begin{aligned}
\int & \left(|H|^{2} \operatorname{div} u_{t}-2 H \cdot \nabla u_{t} \cdot H\right) d x \\
= & \frac{d}{d t} \int\left(|H|^{2} \operatorname{div} u-2 H \cdot \nabla u \cdot H\right) d x \\
& -2 \int\left(H \cdot H_{t} \operatorname{div} u-H_{t} \cdot \nabla u \cdot H-H \cdot \nabla u \cdot H_{t}\right) d x \\
\leq & \frac{d}{d t} \int\left(|H|^{2} \operatorname{div} u-2 H \cdot \nabla u \cdot H\right) d x+C\left\|H_{t}\right\|_{L^{2}}^{2}+C\||H||\nabla u|\|_{L^{2}}^{2} .
\end{aligned}
$$

Substituting (3.16) and (3.20)-(3.24) into (3.13), we obtain after using (3.19) and choosing $\eta$ suitably small that

$$
\begin{aligned}
& \frac{d}{d t} \int \Phi d x+\int \rho|\dot{u}|^{2} d x \\
& \leq C \int|u|^{2}\left(\rho E^{2}+|\nabla u|^{2}+|\nabla v|^{2}\right) d x+C\|\nabla u\|_{L^{2}}^{2} \\
& \quad+C_{2}\left(\|\nabla E\|_{L^{2}}^{2}+\left\|H_{t}\right\|_{L^{2}}^{2}+\|\nabla H\|_{H^{1}}^{2}+\left\|\left|H\|\nabla u \mid\|_{L^{2}}^{2}\right),\right.\right.
\end{aligned}
$$


where

$$
\begin{aligned}
\Phi \triangleq & \mu|\nabla u|^{2}+(\mu+\lambda)(\operatorname{div} u)^{2}-2 P \operatorname{div} u+\mu|\nabla v|^{2}+(\mu+\lambda)(\operatorname{div} v)^{2} \\
& -|H|^{2} \operatorname{div} u+2 H \cdot \nabla u \cdot H
\end{aligned}
$$

satisfies

$$
\Phi \geq \frac{\mu}{2}|\nabla u|^{2}-C_{3} \rho E^{2}-C|H|^{4} .
$$

Next, it follows from (1.1) that for $r, s$ as in (1.7)

$$
\begin{aligned}
& \nu \frac{d}{d t}\|\nabla H\|_{L^{2}}^{2}+\left\|H_{t}\right\|_{L^{2}}^{2}+\nu^{2}\|\Delta H\|_{L^{2}}^{2} \\
& =\int\left|H_{t}-\nu \Delta H\right|^{2} d x \\
& \leq C\left(\||\|H\| \nabla u|\|_{L^{2}}^{2}+\left\|\left|u\|\nabla H \mid\|_{L^{2}}^{2}\right)\right.\right. \\
& \leq C\|H\|_{L^{6}}^{2}\|\nabla u\|_{L^{2}}\|\nabla u\|_{L^{6}}+C\|u\|_{L^{r}}^{2}\|\nabla H\|_{L^{2}}^{2(r-3) / r}\|\nabla H\|_{L^{6}}^{6 / r} \\
& \leq \eta\|\nabla u\|_{L^{6}}^{2}+C(\eta)\|\nabla u\|_{L^{2}}^{2}+C(\varepsilon)\left(1+\|u\|_{L^{r}}^{s}\right)\|\nabla H\|_{L^{2}}^{2}+\varepsilon\left\|\nabla^{2} H\right\|_{L^{2}}^{2} .
\end{aligned}
$$

Noticing that the standard $L^{2}$-estimate of elliptic system gives

$$
\left\|\nabla^{2} H\right\|_{L^{2}} \leq C_{4}\|\Delta H\|_{L^{2}}
$$

after choosing $\varepsilon$ suitably small, we deduce from (3.27) that for any $\eta \in(0,1)$,

$$
\begin{aligned}
& 4 \nu \frac{d}{d t}\|\nabla H\|_{L^{2}}^{2}+4\left\|H_{t}\right\|_{L^{2}}^{2}+2 \nu^{2} C_{4}^{-1}\left(\|\nabla H\|_{H^{1}}^{2}+\|H\| \nabla u \|_{L^{2}}^{2}\right) \\
& \leq C \eta\|\nabla u\|_{L^{6}}^{2}+C(\eta)\|\nabla u\|_{L^{2}}^{2}+C\left(1+\|u\|_{L^{r}}^{s}\right)\|\nabla H\|_{L^{2}}^{2} .
\end{aligned}
$$

Then, adding (3.4) multiplied by $C_{5} \triangleq C_{3} c_{v}^{-1}+\left(C_{2}+2\right) \kappa^{-1}$ and (3.28) by $C_{6} \triangleq$ $\left(1+C_{4} \nu^{-2}\right)\left(C_{2}+C_{1} C_{5}+2\right)$ to (3.25), we obtain that

$$
\begin{aligned}
& \frac{d}{d t} \int\left(\Phi+C_{5} \rho E^{2}+4 C_{6} \nu|\nabla H|^{2}\right) d x \\
& \quad+\|\nabla E\|_{L^{2}}^{2}+\frac{1}{2} \int \rho|\dot{u}|^{2} d x+\left\|H_{t}\right\|_{L^{2}}^{2}+\|\nabla H\|_{H^{1}}^{2} \\
& \leq C \int|u|^{2}\left(\rho E^{2}+|\nabla u|^{2}+|\nabla v|^{2}\right) d x+C\left(1+\|u\|_{L^{r}}^{s}\right)\|\nabla H\|_{L^{2}}^{2} \\
& \quad+C \eta\|\nabla u\|_{L^{6}}^{2}+C(\eta) \int\left(\rho E^{2}+|\nabla u|^{2}\right) d x .
\end{aligned}
$$

Holder's inequality together with (3.15) yields that

$$
\begin{aligned}
& \int|u|^{2}\left(\rho E^{2}+|\nabla u|^{2}+|\nabla v|^{2}\right) d x \\
& \leq C\|u\|_{L^{r}}^{2}\left(\left\|\rho^{1 / 2} E\right\|_{L^{2 r /(r-2)}}^{2}+\|\nabla u\|_{L^{2 r /(r-2)}}^{2}\right) \\
& \leq C\|u\|_{L^{r}}^{2}\left(\left\|\rho^{1 / 2} E\right\|_{L^{2}}^{2(r-3) / r}\|E\|_{L^{6}}^{6 / r}+\|\nabla u\|_{L^{2}}^{2(r-3) / r}\|\nabla u\|_{L^{6}}^{6 / r}\right) \\
& \leq C(\eta)\left(1+\|u\|_{L^{r}}^{s}\right) \int\left(\rho E^{2}+|\nabla u|^{2}\right) d x+\eta\|\nabla E\|_{L^{2}}^{2}+\eta\|\nabla u\|_{L^{6}}^{2},
\end{aligned}
$$


where in the last inequality we have used (3.9). It follows from (3.17), (3.15), (3.19), and (3.9) that

$$
\begin{aligned}
\|\nabla u\|_{L^{6}} & \leq C\|\rho E\|_{L^{6}}+C\|\rho \dot{u}\|_{L^{2}}+C\|\nabla H\|_{L^{2}}^{1 / 2}\|\nabla H\|_{H^{1}}^{1 / 2} \\
& \leq C\left\|\rho^{1 / 2} E\right\|_{L^{2}}+C\|\nabla E\|_{L^{2}}+C\|\rho \dot{u}\|_{L^{2}}+C\|\nabla H\|_{L^{2}}^{1 / 2}\|\nabla H\|_{H^{1}}^{1 / 2} .
\end{aligned}
$$

Putting this and (3.30) into (3.29), and choosing $\eta$ suitably small, we obtain after using Gronwall's inequality, (3.26), (3.1), and (1.26) that

$$
\begin{aligned}
& \sup _{0 \leq t \leq T} \int\left(\rho E^{2}+|\nabla u|^{2}+|\nabla H|^{2}\right) d x \\
& +\int_{0}^{T} \int\left(|\nabla E|^{2}+\rho|\dot{u}|^{2}+|u|^{2}|\nabla u|^{2}+\left|H_{t}\right|^{2}+\left|\nabla^{2} H\right|^{2}\right) d x d t \leq C .
\end{aligned}
$$

Finally, (1.1) $)_{1}$ implies that

$$
(\rho-\tilde{\rho})_{t}+\operatorname{div}((\rho-\tilde{\rho}) u)+\tilde{\rho} \operatorname{div} u=0 .
$$

Multiplying (3.33) by $\rho-\tilde{\rho}$ and integrating the resulting equation over $\Omega$, we obtain after using (1.26) that

$$
\left(\|\rho-\tilde{\rho}\|_{L^{2}}^{2}\right)^{\prime}(t) \leq C\|\rho-\tilde{\rho}\|_{L^{2}}^{2}+C\|\nabla u\|_{L^{2}}^{2},
$$

which together with (3.32) and the following simple fact that

$$
\|\nabla \theta\|_{L^{2}} \leq C\|\nabla E\|_{L^{2}}+C\|\| u\|\nabla u\|_{L^{2}},
$$

directly gives (3.12). The proof of Lemma 3.3 is completed.

Finally, the following Lemma 3.4 will deal with the higher order estimates of the solutions which are needed to guarantee the extension of local strong solution to be a global one under the conditions (1.11)-(1.13) and (1.26).

Lemma 3.4. Under the condition (1.26), it holds that for $0 \leq T<T^{*}$,

$$
\sup _{0 \leq t \leq T}\left(\|\rho-\tilde{\rho}\|_{H^{1} \cap W^{1, \tilde{q}}}+\|\nabla u\|_{H^{1}}+\|\nabla \theta\|_{H^{1}}+\|H\|_{H^{2}}\right) \leq C .
$$

Proof. First, it follows from (3.31), (3.12), and (3.1) that

$$
\begin{aligned}
\|\nabla u\|_{L^{6}} & \leq C+C\|\rho \dot{u}\|_{L^{2}}+C\|\nabla \theta\|_{L^{2}}+C\|\| u\|\nabla u\|\left\|_{L^{2}}+C\right\| \nabla H \|_{H^{1}}^{1 / 2} \\
& \leq C+C\|\rho \dot{u}\|_{L^{2}}+C\|\nabla \theta\|_{L^{2}}+C\|u\|_{L^{6}}\|\nabla u\|_{L^{2}}^{1 / 2}\|\nabla u\|_{L^{6}}^{1 / 2}+C\|\nabla H\|_{H^{1}}^{1 / 2} \\
& \leq C+C\|\rho \dot{u}\|_{L^{2}}+C\|\nabla \theta\|_{L^{2}}+\frac{1}{2}\|\nabla u\|_{L^{6}}+C\|\nabla H\|_{H^{1}}^{1 / 2}
\end{aligned}
$$

which implies

$$
\|\nabla u\|_{L^{6}} \leq C+C\|\rho \dot{u}\|_{L^{2}}+C\|\nabla \theta\|_{L^{2}}+C\|\nabla H\|_{H^{1}}^{1 / 2} .
$$

Then, it follows from the standard $L^{2}$-estimate of (1.1) 4 , (3.12), and (3.1) that

$$
\begin{aligned}
\left\|\nabla^{2} H\right\|_{L^{2}} & \leq C\left\|H_{t}\right\|_{L^{2}}+C\left\|\left|\left\|\nabla H\left|\left\|_{L^{2}}+C\right\|\right| \nabla u\right\| H\right|\right\|_{L^{2}} \\
& \leq C\left\|H_{t}\right\|_{L^{2}}+C\|u\|_{L^{6}}\|\nabla H\|_{L^{2}}^{1 / 2}\left\|\nabla^{2} H\right\|_{L^{2}}^{1 / 2}+C\|H\|_{L^{4}}\|\nabla u\|_{L^{4}} \\
& \leq C\left\|H_{t}\right\|_{L^{2}}+\frac{1}{2}\left\|\nabla^{2} H\right\|_{L^{2}}+C\|\nabla u\|_{L^{4}}+C,
\end{aligned}
$$


which together with (3.12) and (3.1) implies

$$
\|H\|_{H^{2}} \leq C\left\|H_{t}\right\|_{L^{2}}+C\|\nabla u\|_{L^{4}}+C .
$$

Holder's inequality, along with (3.35) and (3.12), gives

$$
\|\nabla u\|_{L^{4}} \leq\|\nabla u\|_{L^{2}}^{1 / 4}\|\nabla u\|_{L^{6}}^{3 / 4} \leq C+C\|\rho \dot{u}\|_{L^{2}}^{3 / 4}+C\|\nabla \theta\|_{L^{2}}^{3 / 4}+C\|\nabla H\|_{H^{1}}^{3 / 8},
$$

which combined with (3.36) and (3.35) shows

$$
\|\nabla u\|_{L^{6}}+\|H\|_{H^{2}} \leq C\|\rho \dot{u}\|_{L^{2}}+C\left\|H_{t}\right\|_{L^{2}}+C\|\nabla \theta\|_{L^{2}}+C .
$$

Then, similar to (3.9), we have

$$
\|\theta\|_{L^{6}} \leq C\left\|\rho^{1 / 2} \theta\right\|_{L^{2}}+C\|\nabla \theta\|_{L^{2}} \leq C+C\|\nabla \theta\|_{L^{2}}
$$

which together with the standard $L^{2}$-estimate of (1.1) 3 and (3.12) gives

$$
\begin{aligned}
& \|\nabla \theta\|_{H^{1}}^{2} \\
& \leq C+C\|\nabla \theta\|_{L^{2}}^{2}+C \int \rho \dot{\theta}^{2} d x+C \int \rho^{2} \theta^{2}|\nabla u|^{2} d x+C\|\nabla u\|_{L^{4}}^{4}+C\|\nabla H\|_{L^{4}}^{4} \\
& \leq C+C\|\nabla \theta\|_{L^{2}}^{2}+C \int \rho \dot{\theta}^{2} d x+C\|\nabla u\|_{L^{2}}^{2}\|\theta\|_{L^{\infty}}^{2}+C\|\nabla u\|_{L^{4}}^{4}+C\|\nabla H\|_{H^{1}}^{4} \\
& \leq C+C \int \rho \dot{\theta}^{2} d x+C\|\nabla \theta\|_{L^{2}}^{2}+\frac{1}{2}\|\nabla \theta\|_{H^{1}}^{2}+C\|\nabla u\|_{L^{4}}^{4}+C\|\nabla H\|_{H^{1}}^{4} .
\end{aligned}
$$

Combining this with (3.36) shows

$$
\|\nabla \theta\|_{H^{1}}^{2} \leq C \int \rho \dot{\theta}^{2} d x+C\|\nabla \theta\|_{L^{2}}^{2}+C\|\nabla u\|_{L^{4}}^{4}+C\left\|H_{t}\right\|_{L^{2}}^{4}+C .
$$

Next, we claim that we have the following estimates on both $\dot{u}$ and $\dot{\theta}$, (3.41) and (3.42), whose proofs are similar to those in [15, 38, and can be found in Appendix A.

$$
\begin{gathered}
\sup _{0 \leq t \leq T} \int\left(|\nabla \theta|^{2}+\rho|\dot{u}|^{2}+\left|H_{t}\right|^{2}\right) d x+\int_{0}^{T} \int\left(\rho \dot{\theta}^{2}+|\nabla \dot{u}|^{2}+\left|\nabla H_{t}\right|^{2}\right) d x d t \leq C, \\
\sup _{0 \leq t \leq T}\left\|\rho^{1 / 2} \dot{\theta}\right\|_{L^{2}}^{2}+\int_{0}^{T}\|\nabla \dot{\theta}\|_{L^{2}}^{2} d t \leq C .
\end{gathered}
$$

Then, the combination of (3.38)-(3.42) with (3.37) leads to

$$
\sup _{0 \leq t \leq T}\left(\|\nabla u\|_{L^{6}}+\|H\|_{H^{2}}+\|\theta\|_{L^{6}}+\|\nabla \theta\|_{H^{1}}\right) \leq C .
$$

For $2 \leq p \leq \tilde{q}$, direct calculations show that

$$
\frac{d}{d t}\|\nabla \rho\|_{L^{p}} \leq C\left(1+\|\nabla u\|_{L^{\infty}}\right)\|\nabla \rho\|_{L^{p}}+C\left\|\nabla^{2} u\right\|_{L^{p}}
$$

For $v \in D_{0}^{1} \cap D^{2,2} \cap D^{2, \tilde{q}}$ satisfying (3.14), it follows from Lemma 2.3 and (3.43) that

$$
\begin{aligned}
\|\nabla v\|_{L^{\infty}} & \leq C\left(1+\log \left(e+\|\nabla(\rho \theta)\|_{L^{\tilde{q}}}\right)\|\rho \theta\|_{L^{\infty}}+\|\rho \theta\|_{L^{2}}\right) \\
& \leq C \log \left(e+\|\nabla \rho\|_{L^{\tilde{q}}}\right) .
\end{aligned}
$$


Then, for $w \triangleq u-v \in D_{0}^{1} \cap D^{2,2} \cap D^{2, \tilde{q}}$ satisfying (3.18), applying the standard $L^{p}$-estimate to (3.18), along with (3.43), gives

$$
\begin{aligned}
\left\|\nabla^{2} w\right\|_{L^{6}} & \leq C\|\rho \dot{u}\|_{L^{6}}+C\left\|\left|H\|\nabla H \mid\|_{L^{6}}\right.\right. \\
& \leq C\|\nabla \dot{u}\|_{L^{2}}+C,
\end{aligned}
$$

which together with (3.19), (3.43), and (3.41) shows

$$
\|\nabla w\|_{L^{\infty}} \leq C+C\|\nabla \dot{u}\|_{L^{2}} .
$$

The combination of this with (3.45) gives

$$
\|\nabla u\|_{L^{\infty}} \leq C \log \left(e+\|\nabla \rho\|_{L^{\tilde{q}}}\right)+C\|\nabla \dot{u}\|_{L^{2}} .
$$

Applying the standard $L^{p}$-estimate to $(1.1)_{2}$ leads to

$$
\begin{aligned}
\left\|\nabla^{2} u\right\|_{L^{p}} & \leq C\left(\|\rho \dot{u}\|_{L^{p}}+\|H\| \nabla H \mid\left\|_{L^{p}}+\right\| \nabla P \|_{L^{p}}\right) \\
& \leq C\left(\|\rho \dot{u}\|_{L^{p}}+\|\nabla \rho\|_{L^{p}}\right)+C \\
& \leq C\left(1+\|\nabla \dot{u}\|_{L^{2}}+\|\nabla \rho\|_{L^{p}}\right)
\end{aligned}
$$

due to (3.41) and (3.43). Substituting (3.47) and (3.46) into (3.44) yields that

$$
f^{\prime}(t) \leq C g(t) f(t) \ln f(t)
$$

where

$$
f(t) \triangleq e+\|\nabla \rho\|_{L^{\tilde{q}}}, g(t) \triangleq 1+\|\nabla \dot{u}\|_{L^{2}} .
$$

It thus follows from (3.48), (3.41), and Gronwall's inequality that

$$
\sup _{0 \leq t \leq T}\|\nabla \rho\|_{L \tilde{q}} \leq C,
$$

which, along with (3.46) and (3.41), directly gives

$$
\int_{0}^{T}\|\nabla u\|_{L^{\infty}}^{2} d t \leq C
$$

Taking $p=2$ in (3.44), we get by using (3.50), (3.47), (3.41), and Gronwall's inequality that

$$
\sup _{0 \leq t \leq T}\|\nabla \rho\|_{L^{2}} \leq C
$$

which together with (3.47), (3.43), and (3.41) yields that

$$
\sup _{0 \leq t \leq T}\left\|\nabla^{2} u\right\|_{L^{2}} \leq C \sup _{0 \leq t \leq T}\left(\|\rho \dot{u}\|_{L^{2}}+\|\nabla \rho\|_{L^{2}}+\|\nabla \theta\|_{L^{2}}+\|H \cdot \nabla H\|_{L^{2}}\right) \leq C .
$$

This combined with (3.49), (3.51), (3.43), and (3.12) finishes the proof of Lemma 3.4.

Now we are in a position to prove Theorem 1.1

Proof of Theorem 1.1. Suppose that (1.14) were false, that is, (1.26) holds. Note that the generic constant $C$ in Lemma 3.4 remains uniformly bounded for all $T<T^{*}$, so the functions $(\rho, u, \theta, H)\left(x, T^{*}\right) \triangleq \lim _{t \rightarrow T^{*}}(\rho, u, \theta, H)(x, t)$ satisfy the conditions imposed 
on the initial data (1.11) at the time $t=T^{*}$. Furthermore, standard arguments yield that $\rho \dot{u}, \rho \dot{\theta} \in C\left([0, T] ; L^{2}\right)$, which implies

$$
(\rho \dot{u}, \rho \dot{\theta})\left(x, T^{*}\right)=\lim _{t \rightarrow T^{*}}(\rho \dot{u}, \rho \dot{\theta}) \in L^{2} .
$$

Hence,

$$
\begin{aligned}
& -\mu \Delta u-(\mu+\lambda) \nabla \operatorname{div} u+R \nabla(\rho \theta)-(\operatorname{curl} H) \times\left. H\right|_{t=T^{*}}=\sqrt{\rho}\left(x, T^{*}\right) g_{1}(x), \\
& \kappa \Delta \theta+\frac{\mu}{2}\left|\nabla u+(\nabla u)^{t r}\right|^{2}+\lambda(\operatorname{div} u)^{2}+\left.\nu|\operatorname{curl} H|^{2}\right|_{t=T^{*}}=\sqrt{\rho}\left(x, T^{*}\right) g_{2}(x),
\end{aligned}
$$

with

$$
g_{1}(x) \triangleq \begin{cases}\rho^{-1 / 2}\left(x, T^{*}\right)(\rho \dot{u})\left(x, T^{*}\right), & \text { for } x \in\left\{x \mid \rho\left(x, T^{*}\right)>0\right\} \\ 0, & \text { for } x \in\left\{x \mid \rho\left(x, T^{*}\right)=0\right\}\end{cases}
$$

and

$$
g_{2}(x) \triangleq \begin{cases}\rho^{-1 / 2}\left(x, T^{*}\right)\left(c_{v} \rho \dot{\theta}+R \rho \theta \operatorname{div} u\right)\left(x, T^{*}\right), & \text { for } x \in\left\{x \mid \rho\left(x, T^{*}\right)>0\right\}, \\ 0, & \text { for } x \in\left\{x \mid \rho\left(x, T^{*}\right)=0\right\},\end{cases}
$$

satisfying $g_{1}, g_{2} \in L^{2}$ due to (3.41), (3.42), and (3.34). Thus, $(\rho, u, \theta, H)\left(x, T^{*}\right)$ also satisfies (1.12) and (1.13). Therefore, one can take $(\rho, u, \theta, H)\left(x, T^{*}\right)$ as the initial data and apply Lemma 2.1 to extend the local strong solution beyond $T^{*}$. This contradicts the assumption on $T^{*}$. We thus finish the proof of Theorem 1.1.

\section{Appendix A Proofs of (3.41) and (3.42).}

The proofs of (3.41) and (3.42) are a direct combination of those of Lemma 4.1 and (4.28) in [15] with that of (3.24) in [38]. We sketch them here for completeness.

First, it follows from (3.12) and (3.38) that

$$
\sup _{0 \leq t \leq T} \int \rho \theta^{2} d x+\int_{0}^{T}\left(\|\nabla \theta\|_{L^{2}}^{2}+\|\nabla u\|_{L^{6}}^{2}\right) d t \leq C .
$$

Applying $\dot{u}_{j}\left[\partial_{t}+\operatorname{div}(u \cdot)\right]$ to $(1.1)_{2}^{j}$ and integrating the resulting equality over $\Omega$ give

$$
\begin{aligned}
\frac{1}{2} \frac{d}{d t} \int \rho|\dot{u}|^{2} d x= & -\int \dot{u}_{j}\left[\partial_{j} P_{t}+\operatorname{div}\left(u \partial_{j} P\right)\right] d x+\mu \int \dot{u}_{j}\left[\partial_{t} \Delta u_{j}+\operatorname{div}\left(u \Delta u_{j}\right)\right] d x \\
& +(\mu+\lambda) \int \dot{u}_{j}\left[\partial_{j} \operatorname{div} u_{t}+\operatorname{div}\left(u \partial_{j} \operatorname{div} u\right)\right] d x \\
& -\frac{1}{2} \int \dot{u}_{j}\left[\partial_{t} \partial_{j}|H|^{2}+\operatorname{div}\left(u \partial_{j}|H|^{2}\right)\right] d x \\
& +\int \dot{u}_{j}\left[\partial_{t} \partial_{i}\left(H^{i} H_{j}\right)+\operatorname{div}\left(u \partial_{i}\left(H^{i} H_{j}\right)\right)\right] d x \\
= & \sum_{i=1}^{5} N_{i} .
\end{aligned}
$$


We get after integration by parts and using the equation (1.1.1) 1 that

$$
\begin{aligned}
N_{1} & =-\int \dot{u}_{j}\left[\partial_{j} P_{t}+\operatorname{div}\left(\partial_{j} P u\right)\right] d x \\
& =R \int \partial_{j} \dot{u}_{j}(\rho \dot{\theta}-\rho u \cdot \nabla \theta-\theta u \cdot \nabla \rho-\theta \rho \operatorname{div} u) d x+\int \partial_{k} \dot{u}_{j} \partial_{j} P u_{k} d x \\
& =R \int \partial_{j} \dot{u}_{j}(\rho \dot{\theta}-\theta \rho \operatorname{div} u) d x+\int P \operatorname{div} \dot{u} \operatorname{div} u d x-\int P \partial_{k} \dot{u}_{j} \partial_{j} u_{k} d x \\
& \leq \frac{\mu}{8}\|\nabla \dot{u}\|_{L^{2}}^{2}+C\|\rho \dot{\theta}\|_{L^{2}}^{2}+C \int \rho^{2} \theta^{2}|\nabla u|^{2} d x \\
& \leq \frac{\mu}{8}\|\nabla \dot{u}\|_{L^{2}}^{2}+C\|\rho \dot{\theta}\|_{L^{2}}^{2}+C\|\rho \theta\|_{L^{2}}^{1 / 2}\|\theta\|_{L^{6}}^{3 / 2}\|\nabla u\|_{L^{4}}^{2} \\
& \leq \frac{\mu}{8}\|\nabla \dot{u}\|_{L^{2}}^{2}+C\|\rho \dot{\theta}\|_{L^{2}}^{2}+C\|\nabla \theta\|_{L^{2}}^{4}+C\|\nabla u\|_{L^{4}}^{4}+C,
\end{aligned}
$$

where in the last inequality we have used (3.39). Integration by parts leads to

$$
\begin{aligned}
N_{2} & =\mu \int \dot{u}_{j}\left[\partial_{t} \triangle u_{j}+\operatorname{div}\left(u \triangle u_{j}\right)\right] d x \\
& =-\mu \int\left(\partial_{i} \dot{u}_{j}\left(\partial_{i} u_{j}\right)_{t}+\triangle u_{j} u \cdot \nabla \dot{u}_{j}\right) d x \\
& =-\mu \int\left(|\nabla \dot{u}|^{2}-\partial_{i} \dot{u}_{j} u_{k} \partial_{k} \partial_{i} u_{j}-\partial_{i} \dot{u}_{j} \partial_{i} u_{k} \partial_{k} u_{j}+\triangle u_{j} u \cdot \nabla \dot{u}_{j}\right) d x \\
& =-\mu \int\left(|\nabla \dot{u}|^{2}+\partial_{i} \dot{u}_{j} \partial_{i} u_{j} \operatorname{div} u-\partial_{i} \dot{u}_{j} \partial_{i} u_{k} \partial_{k} u_{j}-\partial_{i} u_{j} \partial_{i} u_{k} \partial_{k} \dot{u}_{j}\right) d x \\
& \leq-\frac{7 \mu}{8} \int|\nabla \dot{u}|^{2} d x+C \int|\nabla u|^{4} d x
\end{aligned}
$$

Similarly, we have

$$
N_{3} \leq-\frac{7}{8}(\mu+\lambda)\|\operatorname{div} \dot{u}\|_{L^{2}}^{2}+C \int|\nabla u|^{4} d x
$$

Integration by parts together with (3.12) and (3.1) shows

$$
\begin{aligned}
\left|N_{4}\right| & \leq C\|\nabla \dot{u}\|_{L^{2}}\left(\left\|\left|H\left\|H_{t}\left|\left\|_{L^{2}}+\right\|\right| u\right\| H\|\nabla H \mid\|_{L^{2}}\right)\right.\right. \\
& \leq C\|\nabla \dot{u}\|_{L^{2}}\left(\|H\|_{L^{6}}\left\|H_{t}\right\|_{L^{2}}^{1 / 2}\left\|H_{t}\right\|_{L^{6}}^{1 / 2}+\|u\|_{L^{6}}\|H\|_{L^{6}}\|\nabla H\|_{L^{6}}\right) \\
& \leq \varepsilon\|\nabla \dot{u}\|_{L^{2}}^{2}+\eta\left\|\nabla H_{t}\right\|_{L^{2}}^{2}+C(\varepsilon, \eta)\left\|H_{t}\right\|_{L^{2}}^{2}+C(\varepsilon)\left\|\nabla^{2} H\right\|_{L^{2}}^{2} .
\end{aligned}
$$

Similarly, we also have

$$
\left|N_{5}\right| \leq \varepsilon\|\nabla \dot{u}\|_{L^{2}}^{2}+\eta\left\|\nabla H_{t}\right\|_{L^{2}}^{2}+C(\varepsilon, \eta)\left\|H_{t}\right\|_{L^{2}}^{2}+C(\varepsilon)\left\|\nabla^{2} H\right\|_{L^{2}}^{2} .
$$

Substituting (A.3)-(A.7) into (A.2), we obtain after choosing $\varepsilon$ suitably small that

$$
\begin{aligned}
\frac{d}{d t} \int \rho|\dot{u}|^{2} d x+\mu\|\nabla \dot{u}\|_{L^{2}}^{2} \leq & C \int \rho \dot{\theta}^{2} d x+C \eta\left\|\nabla H_{t}\right\|_{L^{2}}^{2}+C(\eta)\left\|H_{t}\right\|_{L^{2}}^{2} \\
& +C\left\|\nabla^{2} H\right\|_{L^{2}}^{2}+C\|\nabla \theta\|_{L^{2}}^{4}+C\|\nabla u\|_{L^{4}}^{4}+C .
\end{aligned}
$$


Next, multiplying (1.1) 3 by $\dot{\theta}$ and integrating the resulting equality over $\Omega$ yield that

$$
\begin{aligned}
& \frac{\kappa}{2}\left(\|\nabla \theta\|_{L^{2}}^{2}\right)_{t}+c_{v} \int \rho|\dot{\theta}|^{2} d x \\
& =-\kappa \int \nabla \theta \cdot \nabla(u \cdot \nabla \theta) d x+\lambda \int(\operatorname{div} u)^{2} \dot{\theta} d x \\
& \quad+2 \mu \int|\mathfrak{D}(u)|^{2} \dot{\theta} d x-R \int \rho \theta \operatorname{div} u \dot{\theta} d x+\nu \int|\operatorname{curl} H|^{2} \dot{\theta} d x \\
& \triangleq \sum_{i=1}^{5} I_{i} .
\end{aligned}
$$

We estimate each $I_{i}(i=1, \cdots, 5)$ as follows:

First, it follows from (3.40) and (3.12) that

$$
\begin{aligned}
\left|I_{1}\right| & \leq C \int|\nabla u \| \nabla \theta|^{2} d x \\
& \leq C\|\nabla u\|_{L^{2}}\|\nabla \theta\|_{L^{2}}^{1 / 2}\|\nabla \theta\|_{L^{6}}^{3 / 2} \\
& \leq \delta\left\|\nabla^{2} \theta\right\|_{L^{2}}^{2}+C(\delta)\|\nabla \theta\|_{L^{2}}^{2} \\
& \leq C \delta \int \rho \dot{\theta}^{2} d x+C(\delta)\|\nabla \theta\|_{L^{2}}^{2}+C\|\nabla u\|_{L^{4}}^{4}+C\left\|H_{t}\right\|_{L^{2}}^{4}+C .
\end{aligned}
$$

Next, integration by parts yields that, for any $\eta \in(0,1]$,

$$
\begin{aligned}
I_{2}= & \lambda \int(\operatorname{div} u)^{2} \theta_{t} d x+\lambda \int(\operatorname{div} u)^{2} u \cdot \nabla \theta d x \\
= & \lambda \int\left((\operatorname{div} u)^{2} \theta\right)_{t} d x-2 \lambda \int \theta \operatorname{div} u \operatorname{div}(\dot{u}-u \cdot \nabla u) d x+\lambda \int(\operatorname{div} u)^{2} u \cdot \nabla \theta d x \\
= & \lambda\left(\int(\operatorname{div} u)^{2} \theta d x\right)_{t}-2 \lambda \int \theta \operatorname{div} u \operatorname{div} \dot{u} d x \\
& +2 \lambda \int \theta \operatorname{div} u \partial_{i} u_{j} \partial_{j} u_{i} d x+\lambda \int u \cdot \nabla\left(\theta(\operatorname{div} u)^{2}\right) d x \\
\leq & \lambda\left(\int(\operatorname{div} u)^{2} \theta d x\right)_{t}+C\|\theta\|_{L^{6}}\|\nabla u\|_{L^{2}}^{1 / 3}\|\nabla u\|_{L^{4}}^{2 / 3}\left(\|\nabla \dot{u}\|_{L^{2}}+\|\nabla u\|_{L^{4}}^{2}\right) \\
\leq & \lambda\left(\int(\operatorname{div} u)^{2} \theta d x\right)_{t}+\eta\|\nabla \dot{u}\|_{L^{2}}^{2}+C(\eta)\|\nabla u\|_{L^{4}}^{4}+C\|\nabla \theta\|_{L^{2}}^{4}+C,
\end{aligned}
$$

where in the last inequality we have used (3.39).

Then, similar to (A.11), we have that, for any $\eta \in(0,1]$,

$$
I_{3} \leq 2 \mu\left(\int|\mathfrak{D}(u)|^{2} \theta d x\right)_{t}+\eta\|\nabla \dot{u}\|_{L^{2}}^{2}+C(\eta)\|\nabla u\|_{L^{4}}^{4}+C\|\nabla \theta\|_{L^{2}}^{4}+C .
$$

Next, it follows from (3.12) and (3.39) that

$$
\begin{aligned}
\left|I_{4}\right| & \leq C\left\|\rho^{1 / 2} \dot{\theta}\right\|_{L^{2}}\left\|\rho^{1 / 2} \theta\right\|_{L^{2}}^{1 / 4}\|\theta\|_{L^{6}}^{3 / 4}\|\nabla u\|_{L^{4}} \\
& \leq \delta \int \rho \dot{\theta}^{2} d x+C(\delta)\|\nabla \theta\|_{L^{2}}^{4}+C\|\nabla u\|_{L^{4}}^{4}+C(\delta),
\end{aligned}
$$


and that

$$
\begin{aligned}
I_{5}= & \nu \int|\operatorname{curl} H|^{2} \theta_{t} d x+\nu \int|\operatorname{curl} H|^{2} u \cdot \nabla \theta d x \\
= & \nu \frac{d}{d t} \int|\operatorname{curl} H|^{2} \theta d x-2 \nu \int \theta \operatorname{curl} H \cdot \operatorname{curl} H_{t} d x+\nu \int|\operatorname{curl} H|^{2} u \cdot \nabla \theta d x \\
\leq & \nu \frac{d}{d t} \int|\operatorname{curl} H|^{2} \theta d x+C\|\theta\|_{L^{6}}\|\nabla H\|_{L^{2}}^{1 / 2}\|\nabla H\|_{L^{6}}^{1 / 2}\left\|\nabla H_{t}\right\|_{L^{2}} \\
& +C\|\nabla H\|_{L^{6}}^{2}\|u\|_{L^{6}}\|\nabla \theta\|_{L^{2}} \\
\leq & \nu \frac{d}{d t} \int|\operatorname{curl} H|^{2} \theta d x+\eta\left\|\nabla H_{t}\right\|_{L^{2}}^{2}+C(\eta)\left(1+\|\nabla \theta\|_{L^{2}}^{2}\right)\left(1+\left\|\nabla^{2} H\right\|_{L^{2}}^{2}\right) .
\end{aligned}
$$

Substituting (A.10)-(A.14) into (A.9), we obtain after choosing $\delta$ suitably small that, for any $\eta \in(0,1]$,

$$
\begin{aligned}
& \frac{d}{d t} \int \Psi d x+c_{v} \int \rho|\dot{\theta}|^{2} d x \\
& \leq C(\eta)\left(1+\|\nabla \theta\|_{L^{2}}^{2}\right)\left(1+\left\|\nabla^{2} H\right\|_{L^{2}}^{2}+\|\nabla \theta\|_{L^{2}}^{2}\right)+C \eta\|\nabla \dot{u}\|_{L^{2}}^{2} \\
& \quad+C \eta\left\|\nabla H_{t}\right\|_{L^{2}}^{2}+C\|\nabla u\|_{L^{4}}^{4}+C\left\|H_{t}\right\|_{L^{2}}^{4}+C,
\end{aligned}
$$

where

$$
\Psi \triangleq \kappa|\nabla \theta|^{2}-2 \theta\left[\lambda(\operatorname{div} u)^{2}+2 \mu|\mathfrak{D}(u)|^{2}+\nu|\operatorname{curl} H|^{2}\right] .
$$

Next, differentiating (1.1) 4 with respect to $t$ and multiplying the resulting equations by $H_{t}$, we obtain after integration by parts and using (3.1) and (3.12) that

$$
\begin{aligned}
& \frac{1}{2} \frac{d}{d t} \int\left|H_{t}\right|^{2} d x+\nu \int\left|\nabla H_{t}\right|^{2} d x \\
& \leq C\left(\left\|\left|u_{t}\left\|H\left|\left\|_{L^{2}}+\right\|\right| u\right\| H_{t}\right|\right\|_{L^{2}}\right)\left\|\nabla H_{t}\right\|_{L^{2}} \\
& \leq C\left(\|\dot{u}\| H\left|\left\|_{L^{2}}+\right\|\right| u\|\nabla u\| H \mid\left\|_{L^{2}}+\right\| u\left\|_{L^{6}}\right\| H_{t}\left\|_{L^{2}}^{1 / 2}\right\| H_{t} \|_{L^{6}}^{1 / 2}\right)\left\|\nabla H_{t}\right\|_{L^{2}} \\
& \leq C\left(\|\dot{u}\|_{L^{6}}\|H\|_{L^{3}}+\|u\|_{L^{6}}\|\nabla u\|_{L^{4}}\|H\|_{L^{12}}+\left\|H_{t}\right\|_{L^{2}}^{1 / 2}\left\|\nabla H_{t}\right\|_{L^{2}}^{1 / 2}\right)\left\|\nabla H_{t}\right\|_{L^{2}} \\
& \leq \frac{\nu}{2}\left\|\nabla H_{t}\right\|_{L^{2}}^{2}+C\|\nabla \dot{u}\|_{L^{2}}^{2}+C\|\nabla u\|_{L^{4}}^{2}+C\left\|H_{t}\right\|_{L^{2}}^{2},
\end{aligned}
$$

which implies

$$
\frac{d}{d t} \int\left|H_{t}\right|^{2} d x+\nu \int\left|\nabla H_{t}\right|^{2} d x \leq C\|\nabla \dot{u}\|_{L^{2}}^{2}+C\|\nabla u\|_{L^{4}}^{2}+C\left\|H_{t}\right\|_{L^{2}}^{2}
$$

Finally, adding (A.8) multiplied by $\eta^{1 / 4}$ and (A.17) by $\eta^{1 / 2}$ to (A.15), we obtain after choosing $\eta$ suitably small and using (3.37) that

$$
\begin{aligned}
& 2 \frac{d}{d t} \int\left(\Psi+\eta^{1 / 2}\left|H_{t}\right|^{2}+\eta^{1 / 4} \rho|\dot{u}|^{2}\right) d x \\
& \quad+\int\left(c_{v} \rho|\dot{\theta}|^{2}+\nu \eta^{1 / 2}\left|\nabla H_{t}\right|^{2}+\mu \eta^{1 / 4}|\nabla \dot{u}|^{2}\right) d x \\
& \leq C(\eta)\left(1+\|\nabla \theta\|_{L^{2}}^{2}\right)\left(1+\left\|\nabla^{2} H\right\|_{L^{2}}^{2}+\|\nabla \theta\|_{L^{2}}^{2}\right) \\
& \quad+C(\eta)\left\|\rho^{1 / 2} \dot{u}\right\|_{L^{2}}^{4}+C(\eta)\left\|H_{t}\right\|_{L^{2}}^{4} .
\end{aligned}
$$


Noticing that (A.16), (3.12), (3.38), and (3.39) lead to

$$
\begin{aligned}
& 2 \int\left(\Psi+\eta^{1 / 2}\left|H_{t}\right|^{2}+\eta^{1 / 4} \rho|\dot{u}|^{2}\right) d x \\
& \geq 2 \kappa\|\nabla \theta\|_{L^{2}}^{2}-C\|\theta\|_{L^{6}}\|\nabla u\|_{L^{2}}^{3 / 2}\|\nabla u\|_{L^{6}}^{1 / 2}-C\|\theta\|_{L^{6}}\|\nabla H\|_{L^{2}}^{3 / 2}\|\nabla H\|_{H^{1}}^{1 / 2} \\
& \quad+2 \int\left(\eta^{1 / 2}\left|H_{t}\right|^{2}+\eta^{1 / 4} \rho|\dot{u}|^{2}\right) d x \\
& \geq \kappa\|\nabla \theta\|_{L^{2}}^{2}+\int\left(\eta^{1 / 2}\left|H_{t}\right|^{2}+\eta^{1 / 4} \rho|\dot{u}|^{2}\right) d x-C(\eta),
\end{aligned}
$$

we directly obtain (3.41) after using Gronwall's inequality, (1.12), (A.18), (3.12), and (A.1).

It remains to prove (3.42). First, it follows from (3.38)-(3.41) that

$$
\sup _{0 \leq t \leq T}\left(\|\theta\|_{L^{6}}+\|\nabla u\|_{L^{2} \cap L^{6}}+\|H\|_{H^{2}}\right)+\int_{0}^{T}\left\|\nabla^{2} \theta\right\|_{L^{2}}^{2} d t \leq C .
$$

Similar to (3.9), we have

$$
\|\dot{\theta}\|_{L^{6}} \leq C\left\|\rho^{1 / 2} \dot{\theta}\right\|_{L^{2}}+C\|\nabla \dot{\theta}\|_{L^{2}} .
$$

Next, applying the operator $\partial_{t}+\operatorname{div}(u \cdot)$ to $(1.1)_{3}$ leads to

$$
\begin{aligned}
c_{v} \rho & \left(\partial_{t} \dot{\theta}+u \cdot \nabla \dot{\theta}\right) \\
= & \kappa \Delta \dot{\theta}+\kappa\left(\operatorname{div} u \Delta \theta-\partial_{i}\left(\partial_{i} u \cdot \nabla \theta\right)-\partial_{i} u \cdot \nabla \partial_{i} \theta\right) \\
& +\left(\lambda(\operatorname{div} u)^{2}+2 \mu|\mathfrak{D}(u)|^{2}\right) \operatorname{div} u+R \rho \theta \partial_{k} u_{l} \partial_{l} u_{k} \\
& -R \rho \dot{\theta} \operatorname{div} u-R \rho \theta \operatorname{div} \dot{u}+2 \lambda\left(\operatorname{div} \dot{u}-\partial_{k} u_{l} \partial_{l} u_{k}\right) \operatorname{div} u \\
& +\mu\left(\partial_{i} u_{j}+\partial_{j} u_{i}\right)\left(\partial_{i} \dot{u}_{j}+\partial_{j} \dot{u}_{i}-\partial_{i} u_{k} \partial_{k} u_{j}-\partial_{j} u_{k} \partial_{k} u_{i}\right) \\
& +\nu\left[\partial_{t}|\operatorname{curl} H|^{2}+\operatorname{div}\left(|\operatorname{curl} H|^{2} u\right)\right] .
\end{aligned}
$$

Multiplying (A.21) by $\dot{\theta}$, we obtain after integration by parts and using (A.19), (3.41), and (A.20) that

$$
\begin{aligned}
& \frac{c_{v}}{2}\left(\int \rho|\dot{\theta}|^{2} d x\right)_{t}+\kappa\|\nabla \dot{\theta}\|_{L^{2}}^{2} \\
& \leq C \int|\nabla u|\left(\left|\nabla^{2} \theta \| \dot{\theta}\right|+|\nabla \theta||\nabla \dot{\theta}|\right) d x+C \int|\nabla u|^{2}|\dot{\theta}|(|\nabla u|+\theta) d x \\
& \quad+C \int \rho|\dot{\theta}|^{2}|\nabla u| d x+C \int \rho \theta|\nabla \dot{u}||\dot{\theta}| d x+C \int|\nabla u\|\nabla \dot{u}\| \dot{\theta}| d x \\
& \quad+C \int\left(\left|\nabla H\left\|\left.\nabla H_{t}|| \dot{\theta}|+| \nabla H\right|^{2}|u \| \nabla \dot{\theta}|\right) d x\right.\right. \\
& \leq C\|\nabla u\|_{L^{3}}\|\nabla \theta\|_{H^{1}}\left(\|\dot{\theta}\|_{L^{6}}+\|\nabla \dot{\theta}\|_{L^{2}}\right)+C\|\nabla u\|_{L^{3}}^{2}\|\dot{\theta}\|_{L^{6}}\left(\|\nabla u\|_{L^{6}}+\|\theta\|_{L^{6}}\right) \\
& \quad+C\|\nabla u\|_{L^{3}}\|\rho \dot{\theta}\|_{L^{2}}\|\dot{\theta}\|_{L^{6}}+C\left\|\rho^{1 / 2} \theta\right\|_{L^{2}}^{1 / 2}\|\theta\|_{L^{6}}^{1 / 2}\|\nabla \dot{u}\|_{L^{2}}\|\dot{\theta}\|_{L^{6}} \\
& \quad+C\|\nabla u\|_{L^{3}}\|\nabla \dot{u}\|_{L^{2}}\|\dot{\theta}\|_{L^{6}}+C\|\nabla H\|_{L^{3}}\left\|\nabla H_{t}\right\|_{L^{2}}\|\dot{\theta}\|_{L^{6}} \\
& \quad+C\|\nabla H\|_{L^{6}}^{2}\|u\|_{L^{6}}\|\nabla \dot{\theta}\|_{L^{2}} \\
& \leq \frac{\kappa}{2}\|\nabla \dot{\theta}\|_{L^{2}}^{2}+C\left\|\nabla^{2} \theta\right\|_{L^{2}}^{2}+C\left\|\rho^{1 / 2} \dot{\theta}\right\|_{L^{2}}^{2}+C\|\nabla \dot{u}\|_{L^{2}}^{2}+C\left\|\nabla H_{t}\right\|_{L^{2}}^{2}+C,
\end{aligned}
$$

which combined with Gronwall's inequality, (1.13), (A.19), and (3.41) directly gives (3.42). 


\section{References}

[1] Agmon, S., Douglis, A., Nirenberg, L.: Estimates near the boundary for solutions of elliptic partial differential equations satisfying general boundary conditions. I, Comm. Pure Appl. Math. 12, 623-727 (1959); II , Comm. Pure Appl. Math. 17, 35-92 (1964)

[2] Beale, J. T., Kato, T., Majda, A.: Remarks on the breakdown of smooth solutions for the 3-D Euler equations, Commun. Math. Phys. 94, 61-66 (1984)

[3] Chen, M., Liu, S.: Blow-up criterion for 3D viscous-resistive compressible magnetohydrodynamic equations, Math. Method. Appl. Sci. DOI: 10.1002/mma.2674

[4] Cho, Y., Kim, H.: Existence results for viscous polytropic fluids with vacuum. J. Differ. Equ. 228, 377-411 (2006)

[5] Ducomet, B., Feireisl, E.: The equations of Magnetohydrodynamics: On the interaction between matter and radiation in the evolution of gaseous stars. Commun. Math. Phys. 226, 595-629 (2006)

[6] Fan, J., Jiang, S., Ou, Y.: A blow-up criterion for compressible viscous heatconductive flows. Annales de l'Institut Henri Poincare (C) Analyse non lineaire, 27, 337-350 (2010)

[7] Fan J., Yu, W.: Strong solution to the compressible magnetohydrodynamic equations with vacuum. Nonlinear Anal. Real World Appl. 10(1), 392-409 (2009)

[8] Feireisl, E.:Dynamics of Viscous Compressible Fluids. Oxford: Oxford University Press, 2004

[9] He, C., Xin, Z.: On the regularity of weak solutions to the magnetohydrodynamic equations. J. Differ. Equ. 213, 235-254 (2005)

[10] Hoff, D.: Discontinuous solutions of the Navier-Stokes equations for multidimensional flows of heat-conducting fluids. Arch. Rational Mech. Anal. 139, 303-354 (1997)

[11] Hu, X.; Wang, D.: Global solutions to the three-dimensional full compressible magnetohydrodynamic flows. Comm. Math. Phys. 283(1), 255-284 (2008)

[12] Haspot, B.: Regularity of weak solutions of the compressible barotropic NavierStokes equations. available at http://arxiv.org/abs/1001.1581.

[13] Huang, X. D.: Some results on blowup of solutions to the compressible NavierStokes equations. PhD Thesis. The Chinese University of Hong Kong, 2009.

[14] Huang, X. D., Li, J.: On breakdown of solutions to the full compressible NavierStokes equations. Methods Appl. Anal. 16(4), 479-490 (2009)

[15] Huang, X. D., Li, J.: Global classical and weak solutions to the threedimensional full compressible Navier-Stokes system with vacuum and large oscillations. http://arxiv.org/abs/1107.4655 
[16] Huang, X. D., Li, J.,Wang, Y.: Serrin-type blowup criterion for full compressible Navier-Stokes system. Arch. Rational Mech. Anal. in press. DOI: 10.1007/s00205$012-0577-5$

[17] Huang, X. D., Li, J., Xin, Z. P.: Serrin type criterion for the three-dimensional viscous compressible flows. Siam J. Math. Anal. 43, 1872-1886 (2011)

[18] Huang, X. D., Li, J., Xin, Z. P.: Blowup criterion for viscous barotropic flows with vacuum states. Comm. Math. Phys. 301, 23-35 (2011)

[19] Huang, X. D., Li, J., Xin, Z. P.: Global well-posedness of classical solutions with large oscillations and vacuum to the three-dimensional isentropic compressible Navier-Stokes equations. Comm. Pure Appl. Math. 65, 549-585 (2012)

[20] Huang, X. D., Xin, Z. P.: A blow-up criterion for classical solutions to the compressible Navier-Stokes equations. Sci. in China 53(3), 671-686 (2010)

[21] Laudau, L.D., Lifshitz, E.M.: Electrodynamics of continuous media. 2nd ed., New York: Pergamon, 1984

[22] Lions, P. L.: Mathematical topics in fluid mechanics. Vol. 2. Compressible models. New York: Oxford University Press, 1998.

[23] Lu, M., Du, Y., Yao, Z. A., et al: A blow-up criterion for compressible MHD equations. Commun. Pure Appl. anal. 11, 1167-1183 (2012)

[24] Matsumura, A., Nishida, T.: The initial value problem for the equations of motion of viscous and heat-conductive gases. J. Math. Kyoto Univ. 20, 67-104 (1980)

[25] Nash, J.: Le problème de Cauchy pour les équations différentielles d'un fluide général. Bull. Soc. Math. France 90 (1962), 487-497.

[26] Nirenberg, L.: On elliptic partial differential equations. Ann. Scuola Norm. Sup. Pisa (3), 13, 115-162 (1959)

[27] Rozanova, O.: Blow up of smooth solutions to the compressible Navier-Stokes equations with the data highly decreasing at infinity. J. Differ. Equ. 245, 1762$1774(2008)$

[28] Serrin, J.: On the uniqueness of compressible fluid motion. Arch. Rational Mech. Anal. 3, 271-288 (1959)

[29] Serrin, J.: On the interior regularity of weak solutions of the Navier-Stokes equations. Arch. Rational Mech. Anal., 9, 187-195 (1962)

[30] Solonnikov, V. A.: General boundary value problems for systems elliptic in the sense of A. Douglis and L. Nirenberg. I , Izv. Akad. Nauk SSSR Ser. Mat., 28(3), 665-706 (1964), English transl., Amer. Math. Soc. Transl. Ser. II 56, 193-232 (1964)

[31] Solonnikov, V. A.: General boundary value problems for Douglis-Nirenberg elliptic systems. II. Proc. Steklov Inst. Math, 92, 269-339 (1968)

[32] Solonnikov, V. A.: On Greens matrices for elliptic boundary problem. I. Proc. Steklov Inst. Math, 110, 123-170 (1970) 
[33] V. A. Solonnikov, On Greens matrices for elliptic boundary problem. II. Proc. Steklov Inst. Math, 116, 187-226 (1971)

[34] Sun, Y. Z., Wang, C., Zhang, Z. F.: A Beale-Kato-Majda Blow-up criterion for the 3-D compressible Navier-Stokes equations. J. Math. Pures Appl., 95, 36-47 (2011)

[35] Sun, Y. Z., Wang, C., Zhang, Z. F.: A Beale-Kato-Majda criterion for three dimensional compressible viscous heat-conductive flows. Arch. Rational Mech. Anal. 201, 727-742 (2011)

[36] Volpert, A. I., Khudiaev, S. I.: On the Cauchy problem for composite systems of nonlinear equations. Mat. Sb. 87, 504-528 (1972)

[37] Xin, Z. P.: Blowup of smooth solutions to the compressible Navier-Stokes equation with compact density. Comm. Pure Appl. Math. 51, 229-240 (1998)

[38] Xu, X. Y., Zhang, J. W: A blow-up criterion for 3D compressible magnetohydrodynamic equations with vaccum. Math. Models Method. Appl. Sci. 22(2), 1150010 (2012) 